\title{
KECK SPECTROSCOPY OF $3<z<7$ FAINT LYMAN BREAK GALAXIES: THE IMPORTANCE OF NEBULAR EMISSION IN UNDERSTANDING THE SPECIFIC STAR FORMATION RATE AND STELLAR MASS DENSITY
}

\author{
Daniel P. Stark ${ }^{1,4}$, Matthew A. Schenker ${ }^{2}$, Richard Ellis $^{2}$, Brant Robertson $^{1}$, Ross McLure $^{3}$, and James Dunlop ${ }^{3}$ \\ ${ }^{1}$ Department of Astronomy, Steward Observatory, University of Arizona, 933 North Cherry Avenue, Rm N204, Tucson, AZ 85721, USA; dpstark@ email.arizona.edu \\ ${ }^{2}$ Cahill Center of Astronomy \& Astrophysics, California Institute of Technology, MS 249-17, Pasadena, CA 91125, USA \\ ${ }^{3}$ Institute for Astronomy, University of Edinburgh, Royal Observatory, Edinburgh EH9 3HJ, UK \\ Received 2012 August 16; accepted 2012 November 19; published 2013 January 17
}

\begin{abstract}
The physical properties inferred from the spectral energy distributions (SEDs) of $z>3$ galaxies have been influential in shaping our understanding of early galaxy formation and the role galaxies may play in cosmic reionization. Of particular importance is the stellar mass density at early times, which represents the integral of earlier star formation. An important puzzle arising from the measurements so far reported is that the specific star formation rates (sSFRs) evolve far less rapidly than expected in most theoretical models. Yet the observations underpinning these results remain very uncertain, owing in part to the possible contamination of rest-optical broadband light from strong nebular emission lines. To quantify the contribution of nebular emission to broadband fluxes, we investigate the SEDs of 92 spectroscopically confirmed galaxies in the redshift range $3.8<z<5.0$ chosen because the $\mathrm{H} \alpha$ line lies within the Spitzer/IRAC $3.6 \mu \mathrm{m}$ filter. We demonstrate that the $3.6 \mu \mathrm{m}$ flux is systematically in excess of that expected from stellar continuum alone, which we derive by fitting the SED with population synthesis models. No such excess is seen in a control sample of spectroscopically confirmed galaxies with $3.1<z<3.6$ in which there is no nebular contamination in the IRAC filters. From the distribution of our $3.6 \mu \mathrm{m}$ flux excesses, we derive an $\mathrm{H} \alpha$ equivalent width distribution and consider the implications for both the derived stellar masses and the sSFR evolution. The mean rest-frame $\mathrm{H} \alpha$ equivalent width we infer at $3.8<z<5.0(270 \AA)$ indicates that nebular emission contributes at least $30 \%$ of the $3.6 \mu \mathrm{m}$ flux and, by implication, nebular emission is likely to have a much greater impact for galaxies with $z \simeq 6-7$ where both warm IRAC filters are contaminated. Via our empirically derived equivalent width distribution, we correct the available stellar mass densities and show that the sSFR evolves more rapidly at $z>4$ than previously thought, supporting up to a $5 \times$ increase between $z \simeq 2$ and 7 . Such a trend is much closer to theoretical expectations. Given our findings, we discuss the prospects for verifying quantitatively the nebular emission line strengths prior to the launch of the James Webb Space Telescope.
\end{abstract}

Key words: galaxies: evolution - galaxies: formation - galaxies: high-redshift - galaxies: starburst - surveys ultraviolet: galaxies

Online-only material: color figures

\section{INTRODUCTION}

Through detailed photometry of Lyman break galaxies (LBGs) undertaken with the Hubble Space Telescope (HST) and the Spitzer Space Telescope, much has been learned regarding the physical properties of galaxies beyond redshift $z \simeq 3$. Stellar masses and star formation rates (SFRs) have now been inferred from broadband photometric spectral energy distributions (SEDs) for thousands of galaxies spanning the redshift range $3<z<7$ (e.g., Egami et al. 2005; Eyles et al. 2005, 2007; Labbé et al. 2006, 2010a, 2010b; Stark et al. 2007, 2009; Ono et al. 2010; González et al. 2010, 2011; Shapley 2011; Lee et al. 2012; Reddy et al. 2012b; Curtis-Lake et al. 2012). The stellar mass density (SMD) derived from these studies has proven a useful integrated constraint on the contribution of galaxies to reionization (e.g., Robertson et al. 2010), while the evolution of physical properties has provided insight into the processes that govern the assembly of early galaxies (e.g., Finlator et al. 2011; Davé et al. 2011, 2012).

A potentially significant puzzle has recently emerged from these studies through measurement of the specific star formation rate $(\mathrm{sSFR})$ at $z>2$. Current observations demonstrate that between $z \simeq 2$ and $z \simeq 7$, the sSFR in galaxies of fixed stellar mass does not evolve strongly (e.g., Stark et al. 2009; González

\footnotetext{
4 Hubble Fellow.
}

et al. 2010), with recent estimates indicating at most a factor of two increase between $z \simeq 2$ and 7 (e.g., Bouwens et al. 2012b; Reddy et al. 2012b). This is in contrast to simple expectations from semi-analytic models and numerical simulations (e.g., Weinmann et al. 2011; Davé et al. 2011, 2012; Dayal \& Ferrara 2012) that predict that the sSFR should closely match the inflow rate of baryonic material. As this mass inflow rate is thought to increase with redshift as $\dot{M} / M \simeq(1+z)^{2.25}$ (Neistein \& Dekel 2008; Dekel et al. 2009), we should expect nearly a $10 \times$ increase in SSFR in galaxies of fixed stellar mass over $2<z<7$, in marked contrast to the observations.

The physical cause of the discrepancy associated with the sSFR evolution remains unclear. As discussed previously (e.g., Bouché et al. 2010; Dutton et al. 2010; Weinmann et al. 2011; Davé et al. 2011, 2012; Reddy et al. 2012b), a plateau in the redshift dependence of the sSFR would suggest that star formation is more inefficient at $z>6$ than at $z \simeq 2$. Various physical processes might be invoked to impede star formation, such as the inefficient formation of molecular hydrogen in low-metallicity galaxies (e.g., Robertson \& Kravtsov 2008; Gnedin et al. 2009; Krumholz \& Dekel 2012) or an increase in the efficiency with which cold gas is removed via large-scale outflows.

Irrespective of any mechanism that might inhibit star formation at early times, it is difficult to reconcile such an inefficiency with the notion that early galaxies provide the ionizing 


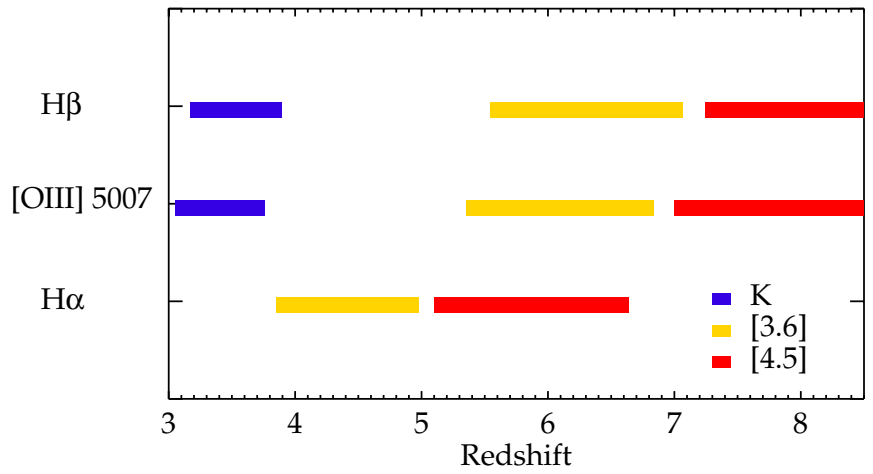

Figure 1. Emission-line contamination of broadband photometry. Colored stripes denote redshift ranges over which emission lines contaminate the $K_{s}$ band (dark blue), IRAC $3.6 \mu \mathrm{m}$ (yellow), and IRAC $4.5 \mu \mathrm{m}$ (red). H $\alpha$ emission is expected in the $3.6 \mu \mathrm{m}$ filter at $3.8<z<5.0$. Note that at $5 \lesssim z \lesssim 7$, both IRAC filters used for measuring stellar masses are contaminated by emission lines. Beyond $z \simeq 7$, only the $4.5 \mu \mathrm{m}$ filter is contaminated by strong nebular emission.

(A color version of this figure is available in the online journal.)

photons responsible for reionization (Robertson et al. 2010). For example, the steep faint-end slope of the ultraviolet luminosity function (UV LF) at $z>6$ (Bouwens et al. 2011b) implies that star formation in low-mass dark matter halos becomes more efficient at earlier times (e.g., Trenti et al. 2010), in contrast to the implications of the sSFR measurements.

Given these difficulties, it is prudent that we reconsider the accuracy of the data that is used to infer the sSFR and its evolution. The two basic ingredients are the SFRs and the stellar masses. The $z>4$ measurements have indeed changed since the original articles (e.g., Stark et al. 2009; González et al. 2010), mostly as a result of improved dust corrections following improved near-infrared photometry (e.g., Bouwens et al. 2012b). The new dust corrections have served to increase the $z \simeq 4 \mathrm{sSFR}$ measurements by a factor of $\simeq 2$. However, since negligible extinction is inferred at $z \simeq 6-7$, the sSFR still remains constant over $4<z<7$ (Bouwens et al. 2012b) although a factor of two higher than at $z \simeq 2-3$ (Reddy et al. 2012b).

A potentially more important problem is the possible contribution of rest-frame optical nebular emission lines (e.g., [O II], [O III], $\mathrm{H} \alpha$ ) to the broadband fluxes used to infer the stellar masses. Such emission lines could significantly affect the inferred amplitude of a Balmer break, leading to an overestimate of the stellar mass and thereby an underestimate of the sSFR. Figure 1 illustrates how the various nebular emission lines contaminate the key photometric filters as a function of redshift. Beyond $z \simeq 4$, the key filters of interest in the determination of stellar masses are the Spitzer/IRAC warm bands at $3.6 \mu \mathrm{m}$ and $4.5 \mu \mathrm{m}$. It is particularly striking that, at $z \gtrsim 5$, the strongest rest-frame optical nebular lines ([O III $] \lambda 5007$ and $\mathrm{H} \alpha$ ) contaminate both Spitzer/IRAC filters. Although many $z \gtrsim 5$ galaxies are detected with Spitzer (e.g., Egami et al. 2005; Eyles et al. 2005; Stark et al. 2009; Labbé et al. 2010a, 2010b; González et al. 2010, 2011; Richard et al. 2011b), contamination by nebular emission could significantly affect the interpretation of their SEDs.

Accounting for nebular emission in the SEDs of high-redshift galaxies has been considered by several earlier works (e.g., Schaerer \& de Barros 2009, 2010; Ono et al. 2010; de Barros et al. 2012). In general terms, their approach has been to use "forward modeling" techniques based on adding nebular emission contributions to stellar population synthesis models in order to demonstrate the possible implications of its inclusion.
However, such "nebular+stellar" model fits cannot provide a precise unambiguous measure of nebular contamination for several reasons. First, there are numerous uncertainties in how the contribution of nebular emission should be added. These include the nebular extinction law and ionizing photon escape fraction. Second, for galaxies at $z>5$, for which there is no uncontaminated measure of the stellar continuum (Figure 1), the uncertainties are particularly large. Finally, and perhaps most importantly, without a spectroscopic redshift, addressing both the nebular contamination and the photometric redshift of the galaxy from the same photometric data leads to great uncertainties; there is no a priori indication of which photometric bands are contaminated by nebular emission.

Fortunately, by virtue of our deep Keck spectroscopic survey (Stark et al. 2010, 2011; Jones et al. 2012; Schenker et al. 2012) and our nebular+stellar population synthesis code (Robertson et al. 2010), we can use the availability of HST-Spitzer SEDs to make progress in addressing this issue. While the question of contamination by nebular emission at $z>5$ must await the infrared spectroscopic capabilities of the James Webb Space Telescope (JWST), we can test our spectroscopic range $3.8<z<5.0$ for contamination by $\mathrm{H} \alpha$ in the Spitzer/IRAC $3.6 \mu \mathrm{m}$ broadband filter. Our approach follows that of Shim et al. (2011), who demonstrated that galaxies in this redshift window are typically significantly brighter at $3.6 \mu \mathrm{m}$ than at $4.5 \mu \mathrm{m}$. By comparing their flux density at $3.6 \mu \mathrm{m}$ to that expected from stellar continuum alone, Shim et al. (2011) argued that many galaxies at $z>4$ show evidence for strong $\mathrm{H} \alpha$ emission, with typical equivalent widths (EWs) significantly greater than those seen at $z \simeq 2$.

Here we seek to apply a similar technique to our spectroscopic sample (Stark et al. 2010, 2011) with the goal of estimating the distribution of $\mathrm{H \alpha} \mathrm{EWs}$ present in galaxies at $3.8<z<5.0$. Equipped with this external constraint on the strength of nebular emission, we can then determine how stellar masses and the sSFR of $z>4$ galaxies are likely to be altered by emissionline contamination. In particular, we will explore whether our estimated degree of nebular contamination could be sufficient at the highest redshifts to permit a rapid rise in the redshiftdependent sSFR as expected from theoretical models.

The present paper is organized as follows. In Section 2, we discuss the selection of the spectroscopic sample used in our analysis. In Section 3, we introduce the details of our SED fitting procedure used to estimate the strength of nebular emission lines in the various filters. In Section 4, we use our spectroscopic sample to estimate the $\mathrm{EW}$ distribution of $\mathrm{H} \alpha$ in the redshift range $3.8<z<5.0$ and then use these measurements to assess the impact of nebular emission on the derived stellar masses and SFRs of $z>4$ galaxies. In Section 5, we discuss the impact that our findings have for the evolution in the integrated SMD and sSFR of galaxies at $z>4$.

Throughout this paper we adopt a $\Lambda$-dominated, flat universe with $\Omega_{\Lambda}=0.7, \Omega_{M}=0.3$, and $H_{0}=70 h_{70} \mathrm{~km} \mathrm{~s}^{-1} \mathrm{Mpc}^{-1}$. All magnitudes are quoted in the AB system (Oke \& Gunn 1983).

\section{DATA}

In this paper, we will focus our analysis on the interpretation of broadband SEDs for a $z>3$ sample with known spectroscopic redshifts. The spectroscopic sample is drawn from earlier papers (Stark et al. 2010, 2011). Full details can be found in these articles, but we offer the reader a brief summary here. Spectroscopy of $z>3$ LBGs in the two GOODS fields was undertaken at the Keck Observatory using the DEIMOS 
spectrograph (Faber et al. 2003). As discussed in Stark et al. (2010), LBGs were selected using standard "dropout" criteria (e.g., Bouwens et al. 2007; Stark et al. 2009) to a limiting magnitude of $z_{850} \simeq 27$ using the GOODS v2 public photometric catalogs (e.g., Giavalisco et al. 2004). Taking advantage of a similar spectroscopic campaign undertaken using the FORS2 in GOODS-South (e.g., Vanzella et al. 2009), we retrospectively constructed a Very Large Telescope (VLT) sample using the same photometric criteria. The combined Keck plus VLT survey comprises 157 galaxies in the redshift range $3.8<z<5.0$ that satisfy the dropout criteria. As we will discuss below, only a subset of these will be used in our analysis.

A key requirement for the derivation of stellar masses and SSFR is precise broadband photometry from which SEDs for galaxies of known spectroscopic redshifts can be determined. In GOODS-South, we use the public release of the Wide Field Camera 3 (WFC3) imaging from the CANDELS MultiCycle Treasury Program (Grogin et al. 2011; Koekemoer et al. 2011) and our own reduction (see McLure et al. 2011) of the Early Release Science campaign (e.g., Windhorst et al. 2011). Colors were computed with respect to the $z_{850}$ flux using matched apertures with up-to-date zero points, and total WFC3 magnitudes were derived by combining the measured colors with the total $z_{850}$-band flux. $K_{s}$-band photometry is taken from deep ISAAC imaging (Retzlaff et al. 2010) following the procedure discussed in Stark et al. (2009). For GOODS-N, we use near-infrared imaging obtained from CFHT/WIRCAM (Wang et al. 2010).

The rest-frame optical at $z>4$ is probed by the deep Spitzer/IRAC (Fazio et al. 2004) imaging of GOODS-S and GOODS-N (M. Dickinson et al. 2012, in preparation). In particular, the $3.6 \mu \mathrm{m}$ (hereafter [3.6]) and $4.5 \mu \mathrm{m}$ (hereafter [4.5]) are the most useful, as the longer wavelength filters are typically not sensitive enough to detect most $z>4$ galaxies. As in Stark et al. (2009), we focus primarily on the subset of ACS-selected galaxies whose IRAC fluxes are not contaminated significantly by neighboring sources. The IRAC magnitudes are measured in apertures 2.4 arcsec in diameter, and to account for flux falling outside this aperture, we apply a 0.7 mag aperture correction derived from a sample of isolated point sources. Recognizing that selecting only isolated IRAC sources limits the size of our eventual sample, we included IRAC flux measurements for galaxies in GOODS-South from the MUSIC catalog (Grazian et al. 2006; Santini et al. 2009). These fluxes rely on a deconfusion procedure to extract fluxes from sources with contaminating neighbors. A comparison between the two photometry methods reveals consistency for our isolated sample, with a standard deviation of 0.19 mag and no systematic offset.

In total, we have 92 galaxies in the range $3.8<z<5.0$ with measured IRAC photometry. In our analysis, we will focus on the subset of 45 galaxies with confident $(>5 \sigma) 4.5 \mu \mathrm{m}$ detections (see Table 1), as without an accurate measure of the $4.5 \mu \mathrm{m}$ flux it is impossible to infer the expected stellar continuum from population synthesis models. The objects with deconfused GOODS MUSIC photometry make up $60 \%$ of the final sample.

Some caution must be exercised when applying inferences from a spectroscopic sample to the parent photometric population. In attempting to infer the typical level of rest-optical nebular contamination, we must be particularly careful that we do not bias our sample toward strong Ly $\alpha$ emitting galaxies, a population that might have larger than average sSFR and $\mathrm{H} \alpha$ EW. For faint galaxies $\left(z_{850}>25.5\right)$, the spectroscopic sample
Table 1

Spectroscopic Sample

\begin{tabular}{|c|c|c|c|c|c|}
\hline ID & R.A. (J2000) & Decl. (J2000) & $z_{\text {spec }}$ & $z 850$ & $\Delta[3.6]$ \\
\hline S44_1649 & 03:32:05.022 & $-27: 46: 12.65$ & 3.91 & 24.47 & 0.07 \\
\hline S43_2212 & $03: 32: 06.615$ & $-27: 47: 47.69$ & 3.94 & 24.28 & 0.13 \\
\hline S33_6294 & $03: 32: 14.497$ & $-27: 49: 32.69$ & 4.74 & 25.40 & 0.26 \\
\hline S33_8715 & $03: 32: 18.257$ & $-27: 48: 02.53$ & 4.28 & 24.65 & 0.45 \\
\hline S33_15763 & $03: 32: 27.939$ & $-27: 46: 18.57$ & 4.00 & 25.23 & 0.17 \\
\hline S23_20730 & $03: 32: 34.349$ & $-27: 48: 55.81$ & 4.14 & 24.11 & 0.22 \\
\hline S24_23979 & $03: 32: 38.729$ & $-27: 44: 13.34$ & 4.00 & 24.81 & 0.00 \\
\hline S23_24940 & $03: 32: 40.086$ & $-27: 49: 01.21$ & 4.13 & 26.45 & 0.35 \\
\hline S24_25118 & $03: 32: 40.385$ & $-27: 44: 31.00$ & 4.13 & 25.24 & 0.04 \\
\hline S22_25614 & 03:32:41.159 & $-27: 51: 01.50$ & 4.06 & 25.25 & 0.42 \\
\hline S23_28451 & $03: 32: 46.247$ & $-27: 48: 46.99$ & 4.02 & 24.88 & 0.39 \\
\hline S12_29436 & $03: 32: 48.244$ & $-27: 51: 36.90$ & 4.36 & 24.87 & 0.67 \\
\hline S13_31908 & $03: 32: 54.035$ & $-27: 50: 00.81$ & 4.43 & 25.07 & -0.25 \\
\hline S12_32366 & $03: 32: 55.249$ & $-27: 50: 22.46$ & 4.17 & 24.42 & 0.20 \\
\hline S12_33166 & $03: 32: 58.380$ & $-27: 53: 39.58$ & 4.40 & 25.75 & 0.14 \\
\hline S43_1669 & 03:32:05.080 & $-27: 46: 56.52$ & 4.82 & 23.79 & 0.14 \\
\hline S44_1745 & $03: 32: 05.259$ & $-27: 43: 00.42$ & 4.80 & 25.24 & 0.14 \\
\hline S45_3792 & $03: 32: 10.027$ & $-27: 41: 32.65$ & 4.81 & 25.03 & 0.27 \\
\hline S34_11180 & $03: 32: 21.931$ & $-27: 45: 33.07$ & 4.79 & 25.82 & 0.17 \\
\hline S33_11861 & $03: 32: 22.884$ & $-27: 47: 27.57$ & 4.44 & 24.93 & 0.43 \\
\hline S33_11915 & $03: 32: 22.971$ & $-27: 46: 29.08$ & 4.50 & 25.34 & 0.33 \\
\hline S35_16226 & $03: 32: 28.563$ & $-27: 40: 55.74$ & 4.60 & 25.44 & 0.24 \\
\hline S31_16819 & 03:32:29.291 & $-27: 56: 19.46$ & 4.76 & 25.05 & 0.10 \\
\hline S22_20041 & $03: 32: 33.475$ & $-27: 50: 30.00$ & 4.90 & 25.77 & 0.25 \\
\hline S24_24961 & 03:32:40.118 & $-27: 45: 35.47$ & 4.77 & 25.55 & 0.45 \\
\hline S21_26522 & $03: 32: 42.623$ & $-27: 54: 28.95$ & 4.40 & 25.61 & 0.58 \\
\hline S12_32900 & $03: 32: 57.169$ & $-27: 51: 45.01$ & 4.76 & 24.64 & 0.40 \\
\hline N33_14884 & $12: 36: 42.235$ & $+62: 15: 22.93$ & 4.42 & 24.47 & 0.27 \\
\hline N14_27206 & $12: 37: 57.510$ & $+62: 17: 18.77$ & 4.71 & 23.82 & 0.28 \\
\hline N42_5352 & $12: 36: 14.513$ & $+62: 11: 40.61$ & 4.15 & 25.31 & 0.30 \\
\hline N42_12760 & $12: 36: 36.823$ & $+62: 12: 04.03$ & 3.90 & 24.94 & 0.35 \\
\hline N33_20202 & $12: 36: 55.940$ & $+62: 14: 12.44$ & 3.91 & 23.78 & 0.15 \\
\hline N33_25472 & $12: 37: 09.840$ & $+62: 14: 39.37$ & 4.25 & 25.01 & 0.32 \\
\hline N23_28987 & $12: 37: 19.688$ & $+62: 15: 42.46$ & 4.53 & 25.49 & 0.27 \\
\hline N34_21578 & $12: 36: 59.377$ & $+62: 19: 05.41$ & 3.86 & 25.30 & 0.10 \\
\hline N34_21756 & $12: 36: 59.758$ & $+62: 18: 54.33$ & 3.86 & 24.78 & 0.27 \\
\hline N34_23754 & $12: 37: 05.013$ & $+62: 17: 31.01$ & 3.93 & 24.62 & 0.31 \\
\hline N35_26133 & $12: 37: 11.814$ & $+62: 22: 12.30$ & 4.05 & 24.25 & 0.27 \\
\hline N35_26600 & $12: 37: 13.037$ & $+62: 21: 11.16$ & 4.05 & 24.09 & 0.33 \\
\hline N24_28740 & $12: 37: 19.003$ & $+62: 19: 53.51$ & 4.19 & 24.38 & 0.18 \\
\hline N25_29248 & $12: 37: 20.446$ & $+62: 22: 14.85$ & 4.05 & 24.63 & 0.58 \\
\hline N24_29391 & $12: 37: 20.845$ & $+62: 18: 43.22$ & 4.07 & 25.42 & 0.41 \\
\hline N32_22884 & $12: 37: 02.520$ & $+62: 11: 55.00$ & 4.02 & 25.64 & 0.10 \\
\hline N24_27374 & $12: 37: 15.103$ & $+62: 20: 05.21$ & 4.06 & 25.85 & 0.35 \\
\hline N42_8958 & $12: 36: 25.972$ & $+62: 08: 59.43$ & 4.14 & 24.36 & 0.49 \\
\hline
\end{tabular}

of Stark et al. (2010) is indeed biased toward Ly $\alpha$ emitters. But by requiring a $5 \sigma$ detection in the [4.5] band, we limit our sample to brighter systems (average $z_{850}$-band magnitude of 25.0) for which we are more complete spectroscopically. Indeed, the percentage of galaxies for which we measure Ly $\alpha$ in emission is actually only $46 \%$, highlighting the fact that many galaxies in this bright subset are instead confirmed via the combination of Ly $\alpha$ in absorption and metal absorption lines (e.g., Jones et al. 2012). Furthermore, the fraction of galaxies in this subset with strong $(\mathrm{EW}>50 \AA$ ) Ly $\alpha$ emission $(5 \%)$ is similar to that measured for galaxies in this $M_{\mathrm{UV}}$ and redshift range $(6 \%$ in Stark et al. 2010), adding confidence that the sample we use in this paper is not likely to be strongly biased toward nebular emitters and appears fairly representative of the photometric population.

It is conceivable that intrinsically fainter galaxies might have larger average nebular EWs than the brighter galaxies that 
we consider. We do not see evidence of strong luminosity dependence within the limited dynamic range of luminosity considered, but if this is the case, it would obviously require even greater downward corrections to the stellar masses. While this likely would not strongly affect our SSFR estimates (which are focused on the more massive and luminous systems at $z \simeq 6$ ), it would impact the faint-end slope of the stellar mass function. Efforts to characterize the luminosity and mass dependence of the nebular EW distribution at $z>4$ are needed to improve measurement of the integrated mass density. Given current Spitzer sensitivity limits, this is likely only feasible via examination of lensed galaxies in the same redshift range considered in this paper.

\section{POPULATION SYNTHESIS MODELING}

\subsection{Modeling Procedure}

Our goal is to quantify the nebular contribution through analysis of the SEDs of a large spectroscopic sample at $3.8<z<5.0$. The advantage of our technique is that, for these sources, we can predict the exact wavelengths of rest-frame optical emission lines and thereby remove ambiguities associated with determining the photometric redshift simultaneously from contaminated broadband photometry.

Previous attempts to assess the impact of nebular emission on broadband photometry have utilized models that include the contributions from both nebular and stellar emission. For the reasons outlined in Section 1, our analysis will focus instead on models containing only stellar continuum. The stellar continuum predictions are based on the models of Bruzual \& Charlot (2003), and the technique we will adopt is mostly similar to that described in detail in Stark et al (2009). However, we also investigate how including nebular emission affects the derived physical properties. To do so, we make use of the code described in Robertson et al. (2010), to which the interested reader is referred. In this code, line emission is calculated from the number of ionizing photons per second, which is provided as output from our population synthesis models.

In the Robertson et al. (2010) code, the intensities of hydrogen lines are computed from the values tabulated in Osterbrock $\&$ Ferland (2006), assuming case B recombination. We compute the intensities of the lines of common metallic species from the empirical results of Anders \& Fritze-v. Alvensleben (2003), assuming a gas-phase metallicity of $Z=0.2 Z_{\odot}$, similar to that measured for galaxies at these redshifts (Maiolino et al. 2008; Jones et al. 2012). The recombination-line luminosities are calculated assuming that the ionizing photon escape fraction, $f_{\text {esc }}$, is 0.2 (e.g., Vanzella et al. 2010; Nestor et al. 2011). Since we do not rely on the nebular models for our primary conclusions, this assumption does not affect our results. The continuum contribution from bound-free, free-free, and two-photon continuum emission is also calculated following Osterbrock \& Ferland (2006). The full nebular template is then added to each stellar continuum model, which is then used to calculate the synthetic fluxes used in our SED fitting code.

Since our sample has the virtue of precise spectroscopic redshifts, we do not fit the photometric bands spanning the Ly $\alpha$ forest and $\operatorname{Ly} \alpha$ emission lines, both of which vary significantly from source to source at any given redshift. For the redshift range that we are primarily interested in $(3.8<z<5.0)$, this leaves seven to eight (largely) independent photometric constraints on the SED in GOODS-S and six constraints on GOODS-N.
For consistency with the earlier literature, we consider a Salpeter (1955) initial mass function with $0.1-100 M_{\odot}$. Given the relatively small number of constraints on the SED, we utilize a moderately restricted grid, varying only the age, dust reddening, and normalization factor. We fix the star formation history $(\mathrm{SFH})$ as either constant or rising with time following the $t^{1.7}$ power law inferred in Papovich et al. (2011). This restricted grid of SFH is supported by the results of Reddy et al. (2012b) that demonstrate that at $z \simeq 2$, the SFRs inferred from exponentially declining SFH models do not agree with those measured from the observed IR and UV fluxes. Nevertheless, we have verified that our results would not be affected if we had adopted exponential decay models. Finally, we utilize sub-solar metallicity $\left(Z=0.2 Z_{\odot}\right)$ motivated by the observations discussed above.

We allow the differential extinction, $E(B-V)_{\text {stars }}$, to range between 0.00 and 0.50 in steps of 0.02 , and we limit the model ages to lie between $5 \mathrm{Myr}$ and the age of the universe at the redshift of interest. The precise form of the dust attenuation curve is, of course, not known at $z>4$, but we consider the reddening law appropriate for local starbursts (e.g., Meurer et al. 1999; Calzetti et al. 2000) and a steeper attenuation curve that is appropriate for the Small Magellanic Cloud (e.g.,Gordon \& Clayton 1998). The latter appears to be appropriate for young galaxies ( $<100 \mathrm{Myr}$ ) at high redshift (Siana et al. 2008; Reddy et al. 2010, 2012a), a population that might become increasingly dominant at $z>4$.

The relative extinction provided to stars and nebular emission is not definitively understood at high redshift. Expectations from nearby galaxies suggest that the nebular gas is preferentially more extincted than the stellar continuum, as expected if the $\mathrm{H}$ II regions lie in dustier regions than the stars contributing to the integrated stellar continuum. Based on observations of local starforming galaxies and starbursts, Calzetti et al. (2000) suggest that $A_{\mathrm{V} \text {,neb }}=A_{\mathrm{V}, \mathrm{SED}} / 0.44$. Whether or not this relationship holds at high redshift is unclear. Some of the first studies of $\mathrm{H} \alpha$ emission in star-forming galaxies $z \simeq 2$ indicated that the nebular gas and stars might be equally attenuated (e.g., Erb et al. 2006; Reddy et al. 2010). But more recently, new studies have emerged that support a factor of $\simeq 2$ higher extinction toward $\mathrm{H}$ II regions (e.g., Förster-Schreiber et al. 2009; Onodera et al. 2010; Mancini et al. 2011; Wuyts et al. 2011), similar to that observed locally. Clearly an improved understanding of how the relative distribution of stars and $\mathrm{H}$ II regions depends on age and mass would greatly benefit attempts to simultaneously fit stellar and nebular emission via population synthesis models. In the nebular+stellar models presented in this paper, we will simply assume that $A_{\mathrm{V}, \mathrm{SED}}=A_{\mathrm{V}, \mathrm{neb}}$.

In the following, we will fit the data with both the nebular+stellar and stellar continuum models. For the latter, the cleanest method is obtained by fitting the data excluding the [3.6] flux measurement, given that this band could be contaminated by $\mathrm{H} \alpha$. However, excluding this band means that only one filter is available to constrain the SED beyond the Balmer break. We therefore also fit the data using all photometric information, i.e., including the [3.6] measurement. This is discussed further in Section 3.2. For each galaxy, we compute the model age, normalization, and $A_{\mathrm{V}}$ that provide acceptable fits to the data. The normalization is then mapped to the SFR and stellar mass appropriate for the given template. We compute uncertainties on these parameters by bootstrap resampling the data within the allowed photometric uncertainties. 

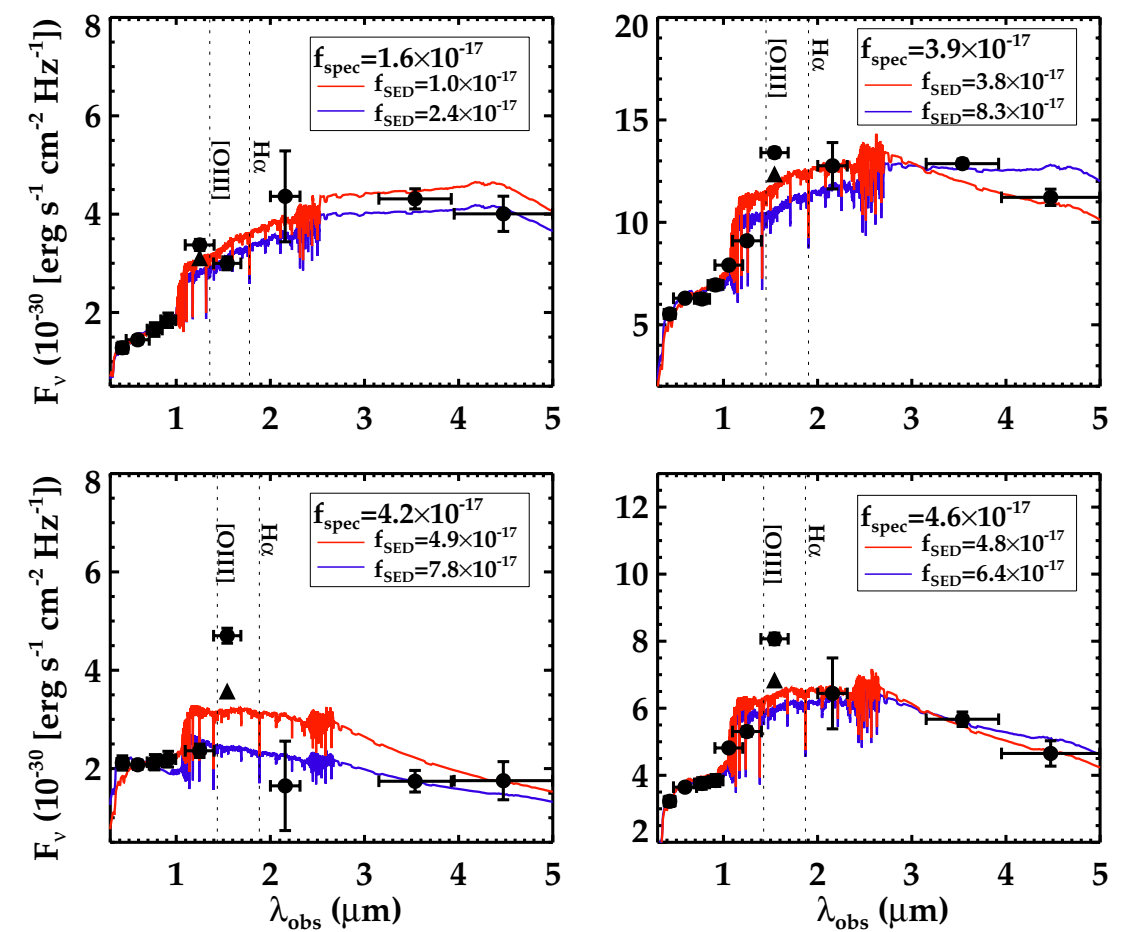

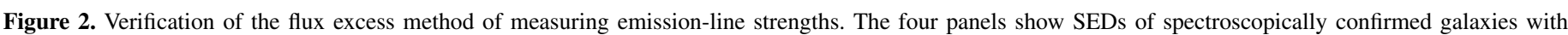

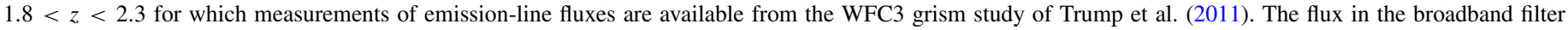

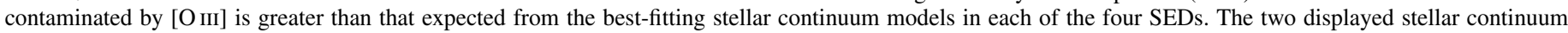

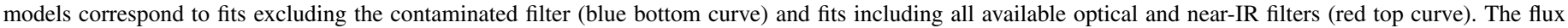

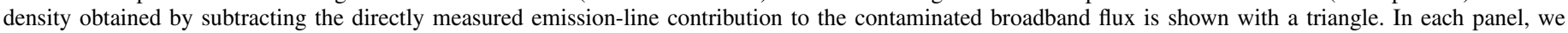

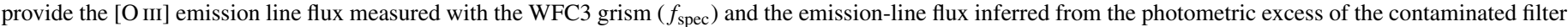
with respect to the stellar continuum models $\left(f_{\mathrm{SED}}\right)$ in units of erg $\mathrm{cm}^{-2} \mathrm{~s}^{-1}$.

(A color version of this figure is available in the online journal.)

\subsection{Nebular Line Strengths}

We infer $\mathrm{H} \alpha$ emission line strengths in our sample of $3.8<$ $z<5.0$ galaxies by comparing the observed flux density in the [3.6] bandpass to the flux density in that filter expected from stellar continuum alone. We explore the method that produces the most accurate EWs below.

To verify the reliability of using the broadband flux excesses to derive emission-line strengths, we examine the SEDs of a moderate-redshift sample of nebular line emitters with spectroscopically measured [O III] line fluxes from WFC3 grism observations of the Hubble Ultra Deep Field (UDF; Trump et al. 2011). We choose this sample rather than larger ground-based samples because use of two-dimensional grism spectroscopy avoids uncertainties owing to slit losses. To characterize the contribution of the emission lines to the broadband SEDs, we measure optical through mid-IR photometry using the UDF data set (see McLure et al. 2011 for details) and perform population synthesis modeling following exactly the same procedure as described above. [O III] will fall in either the $J_{125}$ band (at $1.3<z<1.8$ ) or $H_{160}$ band (at $1.8<z<2.3$ ). We characterize the likely strength of the emission lines by comparing the observed broadband flux (in the contaminated filter) to the stellar continuum flux expected from the best-fitting population synthesis models. We consider the stellar models with and without the contaminated bandpass included. In order to ensure a reliable measure of the stellar continuum in the vicinity of [O III], we do not consider galaxies with strong emission lines in adjacent infrared filters (e.g., [O III] in $H_{160}$ and $\mathrm{H} \alpha$ in $K_{s}$ ) or those undetected in $K_{s}$ band.
We focus our analysis on the four systems in this remaining subset for which the measured [O III] flux is predicted to make a significant (e.g., $\gtrsim 4 \%$ ) contribution to the broadband photometry. In each of these systems, the contaminated filter reveals an excess with respect to the best-fitting stellar continuum model (Figure 2). The fluxes required to produce the broadband excesses in the contaminated filter agree well with those measured spectroscopically (Figure 2), with the results from the two fitting methods typically bracketing the observed line flux. While both methods produce remarkably good agreement, the line fluxes are slightly more accurate (average flux uncertainty of $\simeq 20 \%$ ) when the stellar continuum is estimated from the fit to the SED including the contaminated filter. When the contaminated filter is excluded from the fitting process, the inferred line flux is typically $1.5-2.0 \times$ greater than measured with the WFC3 grism. Given that the SEDs are fairly poorly sampled in the wavelength range where the continuum flux is required, it is conceivable that by excluding the contaminated filter, the fitting process will prefer redder models that fit the flux in the adjacent filters but underpredict the continuum in the vicinity of the emission line of interest. Regardless of the precise reason, it is clear from Figure 2 that by considering the results from both methods, fairly accurate line strengths can be extracted from the photometry.

The results of this test therefore motivate use of the broadband flux excesses to infer line strengths in carefully selected spectroscopic samples at higher redshifts where direct spectroscopic measurements of nebular line fluxes are not yet available. As a result of the higher redshifts (and the corresponding $1+z$ boost in observed EW), we expect the nebular line contribution to broadband fluxes to be greater than typically observed 


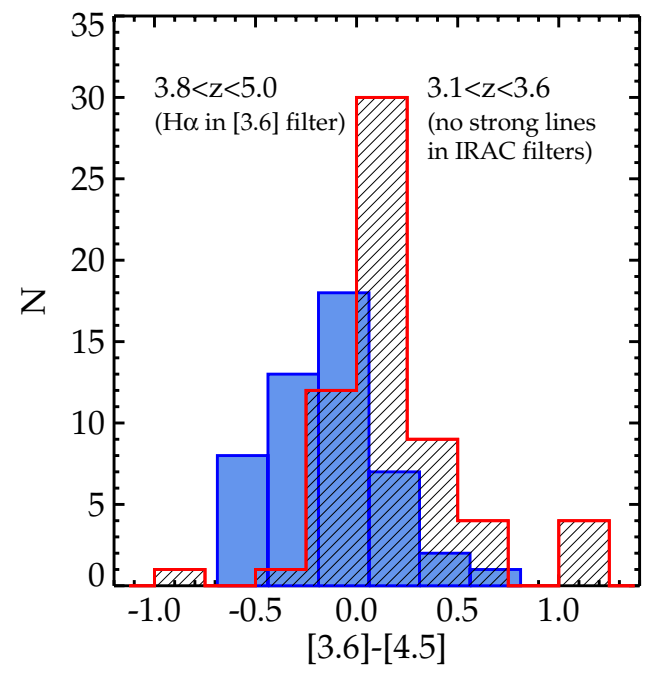

Figure 3. Effect of emission lines on broadband colors. The distribution of [3.6] - [4.5] colors for galaxies at $3.8<z<5.0$ (blue filled histogram) and at $3.1<z<3.6$ (red shaded histogram). The colors in the lower redshift sample reflect the reddened stellar continuum, as both filters are free from strong emission lines. In contrast, the colors of the $3.8<z<5.0$ galaxies are shifted toward bluer values by $\mathrm{H} \alpha$ emission (which lies in the $3.6 \mu \mathrm{m}$ bandpass).

(A color version of this figure is available in the online journal.)

at intermediate redshifts (e.g., Trump et al. 2011), allowing the flux excesses to more consistently stand out with respect to photometric uncertainties. Based on the results in this section, we expect the true line fluxes to be close to our two fitting methods, with the most accurate measurements obtained by fits to the entire SED.

\section{RESULTS}

We now discuss the results derived from applying our technique to the SEDs of spectroscopically confirmed galaxies in the redshift range $3.8<z<5.0$, over which $\mathrm{H} \alpha$ may contaminate the IRAC $3.6 \mu \mathrm{m}$ filter. We compare the observed [3.6] flux densities to the stellar continuum expected from population synthesis models and use the results to infer an empirically based $\mathrm{H} \alpha \mathrm{EW}$ distribution (Section 4.1). We discuss the possible redshift evolution of the nebular line strengths in Section 4.2. Using the empirically derived EW distributions, we examine how nebular emission affects the derived physical properties of $z>4$ galaxies (Section 4.3).

\subsection{Strength of Nebular Emission Lines}

We begin by comparing the [3.6] - [4.5] color distribution for galaxies at $3.8<z<5.0$ with that for galaxies at $3.1<z<3.6$ (a redshift range over which the IRAC colors are uncontaminated by strong nebular emission). This should reveal the impact of $\mathrm{H} \alpha$ emission on broadband fluxes at $z>3$. We apply this test for 45 galaxies at $3.8<z<5.0$ with robust flux measurements in the $4.5 \mu \mathrm{m}$ filter, which constrains the rest-optical stellar continuum. The results (Figure 3) point to a significant contribution from $\mathrm{H} \alpha$. The median [3.6] - [4.5] color at $3.8<z<5.0$ is 0.33 mag bluer than the median value at $3.1<z<3.6$, consistent with expectations if $\mathrm{H} \alpha$ pollutes the $3.6 \mu \mathrm{m}$ filter in the higher redshift bin. Note that the slightly red [3.6] $-[4.5]$ colors of the $3.1<z<3.6$ sample are exactly what is expected for moderately reddened $(E[B-V] \simeq$ $0.1)$ galaxies with a constant SFH and luminosity-weighted ages of 100 Myr. A Kolmogorov-Smirnov test demonstrates with confidence that these two color distributions are distinct (K-S statistic of $D=0.54$ with an associated probability by chance of $8 \times 10^{-8}$ ). We also consider whether the change in color might be due to photometric scatter from increased photometric error in the higher redshift bin. We test this by randomly perturbing the $3.1<z<3.6$ [3.6] - [4.5] color distribution according to the IRAC flux errors of the $3.8<z<5.0$ sample. While this can slightly broaden the width of the color distribution, it does not shift the median color to bluer values as observed.

While the most natural interpretation of the systematic offset is the presence of $\mathrm{H} \alpha$ in the [3.6] filter, it is conceivable that other effects could contribute. For example, one might expect that a systematic offset in [3.6] - [4.5] colors might arise from the slightly different rest-frame wavelengths sampled and the (potentially) younger ages in the higher redshift bin. Examination of population synthesis models indicates that intrinsic galaxy evolution is not likely to dominate the shift in [3.6] - [4.5] colors. Given the median reddening and ages inferred for the $3.1<z<3.6$ and $3.8<z<5.0$ spectroscopic samples, we would expect to see [3.6] $-[4.5] \simeq 0.1$. This is similar to that observed at $3.1<z<3.6$, but significantly redder than that observed in the $3.8<z<5.0$ redshift range with $\mathrm{H} \alpha$ contamination. We therefore conclude that nebular contamination is likely the dominant cause of the differences in the [3.6] - [4.5] colors.

A particularly convincing verification of the above statistical test is the fact that we can directly see evidence of strong nebular emission in individual SEDs (Figure 4). Clearly in these examples the flux in the $3.6 \mu \mathrm{m}$ filter is not only in excess of that at $4.5 \mu \mathrm{m}$ but also significantly in excess of the stellar continuum of the best-fitting population synthesis models. The SEDs of galaxies in this redshift range are (not surprisingly) typically better fit by models including nebular emission (blue lines in bottom panel, see red lines in top panel for stellar continuum models in Figure 4).

To estimate the strength of $\mathrm{H} \alpha$, we compute the amount by which the observed $3.6 \mu \mathrm{m}$ flux exceeds the predicted stellar continuum flux. We define the $3.6 \mu \mathrm{m}$ excess, $\Delta[3.6]$, as the difference between the [3.6] magnitude expected from stellar continuum models that fit the SED and the observed [3.6] magnitude. Positive values indicate that the observed flux is greater than can be accommodated by stellar continuum. This test requires an accurate measure of the stellar continuum in the rest-optical. Again we limit our sample to those galaxies with confident [4.5] detections, as this filter (devoid of strong emission lines) is necessary to anchor the population synthesis models beyond the Balmer break.

The distribution of $3.6 \mu \mathrm{m}$ magnitude excesses in our spectroscopic sample (Figure 5) reveals that $96 \%$ of galaxies are observed to be brighter at [3.6] than predicted from the best-fitting stellar continuum models. The median excess, 0.27 mag, suggests that the typical rest-frame emission line EW in the $3.6 \mu \mathrm{m}$ filter at $3.8<z<5.0$ is $360-450 \AA$. Emission lines therefore contribute nearly $30 \%$ of the observed [3.6] broadband photometry. These values are derived from stellar continuum model fits that include the contaminated $3.6 \mu \mathrm{m}$ filter in the modeling. As we demonstrated in Section 3, this method produces the most accurate flux estimates. If the contaminated filter is excluded from the modeling procedure, the inferred continuum level is typically reduced, resulting in a slightly larger median [3.6] excess $(0.37 \mathrm{mag})$ and total EWs $(520-650 \AA)$. Note that these represent the EWs of all emission lines in the [3.6] filter. We 


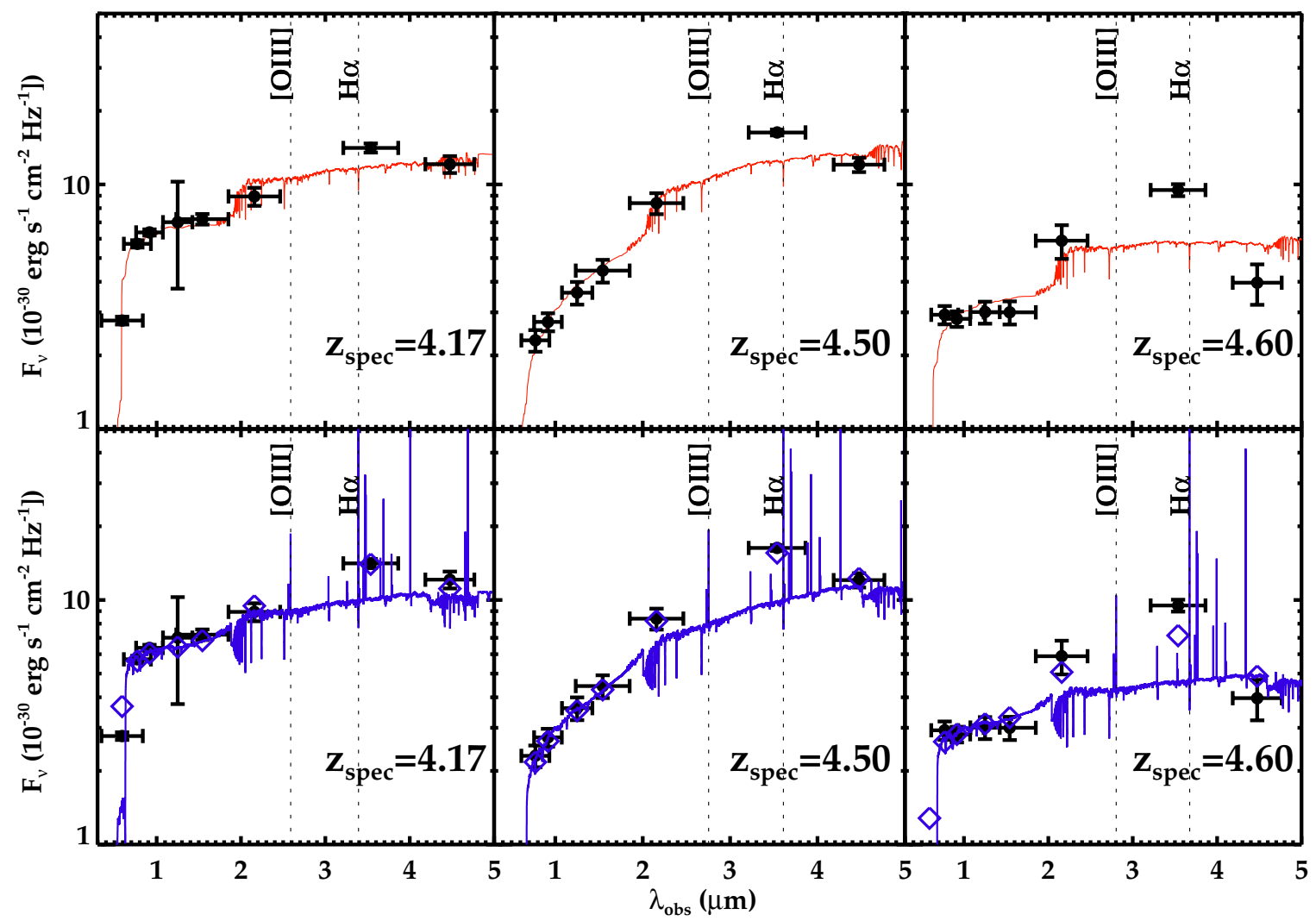

Figure 4. SEDs of spectroscopically confirmed galaxies at $3.8<z<5.0$ fit using population synthesis models containing both stellar continuum (top row) and stellar+nebular emission (bottom row). Many galaxies in this redshift range show blue [3.6] - [4.5] colors, with the $3.6 \mu \mathrm{m}$ flux significantly in excess of the best-fitting stellar continuum. This flux excess is strongly suggestive of $\mathrm{H} \alpha$ nebular line contamination. Not surprisingly, models containing nebular emission provide significantly better fits to the observed photometry, as clearly indicated by the agreement between the synthetic (open blue diamonds) and observed (solid black circles) in the bottom row. In the following, we will only consider objects for which the Balmer break can be anchored by a significant $(\mathrm{S} / \mathrm{N}>5)[4.5]$ detection, removing fainter objects (like that in the right panel) for which an accurate flux excess is difficult to extract.

(A color version of this figure is available in the online journal.)

derive the fractional contribution of $\mathrm{H} \alpha$ below. Based on the discussion in Section 3.2, it is likely that these measurements bracket the range of mean nebular emission line strengths in $3.8<z<5.0$ galaxies. Reassuringly, this EW range is consistent with that required to explain the 0.33 mag offset in median [3.6] - [4.5] colors of $3.1<z<3.6$ and $3.8<z<5.0$ galaxies in Figure 3.

To estimate the EW distribution of emission lines contaminating the IRAC $3.6 \mu \mathrm{m}$ filter, we need to admit a range of EWs to reproduce the observed $3.6 \mu \mathrm{m}$ photometric excess distribution of the top panel of Figure 5. We assume that EWs are distributed in a lognormal fashion, similar to that seen from $\mathrm{H} \alpha$ emission locally and at moderate redshifts (e.g., Lee et al. 2007, 2012; Ly et al. 2011). We consider a large grid spanning a range of $\sigma$ and $\mu$, the width and mean of the EW distribution. We translate each $\mathrm{EW}$ into a $3.6 \mu \mathrm{m}$ excess, applying a photometric scatter of $20 \%$ (a conservative estimate for the average $3.6 \mu \mathrm{m}$ magnitude error) and compute the flux excess distribution expected from the input EW distribution. We find that the observed flux excess distribution is well fit by an EW distribution with $\sigma=0.25$ and $\left\langle\log _{10}\left(W_{[3.6]} / \AA\right)\right\rangle=2.57$ (Figure 5, bottom panel). This EW will surely be dominated by $\mathrm{H} \alpha$ emission, but other emission lines ([S II], [N II]) may of course contribute. The contribution of other lines will depend on the physical properties (e.g., metallicity) of the galaxies. Our sub-solar $\left(0.2 Z_{\odot}\right)$ metallicity models indicate that $\mathrm{H} \alpha$ should contribute $\simeq 76 \%$ of the observed EW. In this case, the typical $\mathrm{H} \alpha \mathrm{EW}$ at $3.8<z<5.0$ is $\left\langle\log _{10}\left(W_{\mathrm{H} \alpha} / \AA\right)\right\rangle=2.45$. With these assumptions, if the [3.6] filter is excluded from the fitting, we find $\left\langle\log _{10}\left(W_{\mathrm{H} \alpha} / \AA\right)\right\rangle=2.61$. As above, we adopt this as an upper bound to the average $\mathrm{H} \alpha \mathrm{EW}$. We note that the width of the distribution we infer at $3.8<z<5.0$ is very similar to the $\sigma=0.3$ derived at $z=2.2$ (Lee et al. 2012).

The level of $\mathrm{H} \alpha$ emission quoted above is actually very reasonable given the typical properties of $z \simeq 4-5$ LBGs (e.g., Stark et al. 2009; González et al. 2011). For constant star formation, ionizing photon escape fraction of 0.2 , and ages of $\simeq 100-250 \mathrm{Myr}$, we would expect $\mathrm{H} \alpha$ EWs to be $\simeq 200-300 \AA$, similar to the range we infer. So the observation of [3.6] excesses of $0.2-0.3$ mag relative to stellar continuum (Figure 3) is exactly what we would expect given the shape of the overall SEDs. Indeed, the absence of any nebular contamination at $3.8<z<5.0$ would have been a far more surprising finding.

\subsection{Evolution of Nebular EW Distribution}

Before evaluating how nebular emission affects the derived stellar masses at $4<z<7$, it is interesting to consider whether the nebular EW distribution is likely to evolve with redshift. Fumagalli et al. (2012) recently examined the evolution of $\mathrm{H} \alpha$ EWs at lower redshifts, finding that the evolution could be fit by a power law $\propto(1+z)^{1.8}$ for galaxies with $10^{10} M_{\odot}$ in stellar mass (Figure 6). While our sample size is too modest to permit a detailed comparison with this trend at $z \gtrsim 4$, it is of interest to consider how the EWs we derived in Section 4.1 compare to those at lower redshift. We note that the $\mathrm{H} \alpha$ EWs presented in Fumagalli et al. (2012) include the contribution of [N II], while 

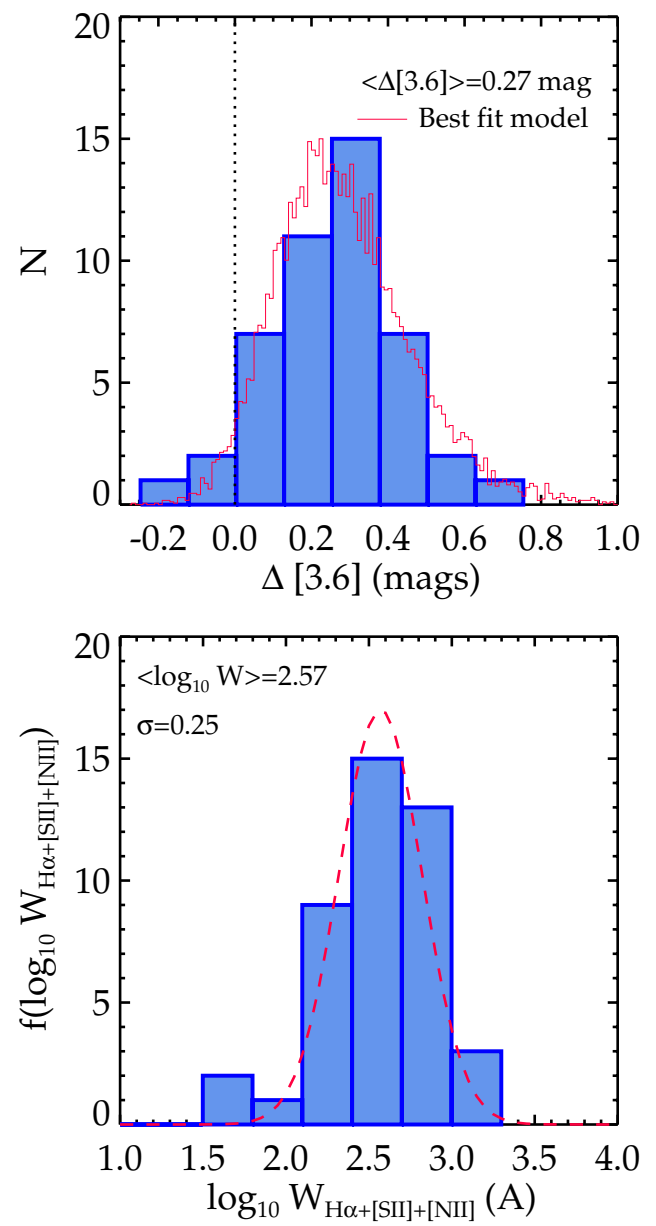

Figure 5. Top: the distribution of $3.6 \mu \mathrm{m}$ magnitude excesses $(\Delta[3.6])$ in our $3.8<z<5.0$ galaxy sample. The magnitude excess is defined as the difference between the [3.6] magnitude inferred from the stellar continuum of the best-fitting population synthesis model and the [3.6] magnitude observed with Spitzer/IRAC. The positive $\Delta[3.6]$ values exhibited by our sample indicate that the stellar continuum is unable to account for the observed flux in the $3.6 \mu \mathrm{m}$ filter. The magnitude excess distribution is well fit by a lognormal equivalent width distribution with $\left\langle\log _{10} W\right\rangle=2.57$ and $\sigma=0.25$ (red curve). Bottom: distribution of equivalent widths required to reproduce the observed flux excesses (blue histogram) compared to the functional form we adopt for the equivalent width distribution (red curve).

(A color version of this figure is available in the online journal.)

the nebular line strengths we infer from photometric excesses include the contribution of all emission lines contaminating the [3.6] filter. Using our $0.2 Z_{\odot}$ population synthesis models, we estimate that $82 \%$ of the EW inferred from [3.6] photometric excesses arises from $\mathrm{H} \alpha$ and $[\mathrm{N} I I]$. Note that this is slightly larger than the percentage estimated in the previous section owing to the addition of $[\mathrm{N}$ II] to the calculation.

Applying this factor to the mean EWs presented in Section 4.1, we compare the $\mathrm{H} \alpha+[\mathrm{N}$ II] EWs at $3.8<z<5.0$ to those at $z \lesssim 2$ (Figure 6 ). It is clear that the line strengths derived at $3.8<z<5.0$ are consistent with a continued increase in the $\mathrm{H} \alpha \mathrm{EW}$ in the range $2 \lesssim z \lesssim 5$. While determination of the exact rate of increase is beyond the scope of this work, we note that the $\mathrm{H} \alpha$ EWs at $3.8<z<5.0$ lie in the range expected by simple extrapolations of the power laws derived in Fumagalli et al. (2012). This increase in $\mathrm{H} \alpha \mathrm{EW}$ over $2<z<5$, albeit tentative in nature, is supportive of an increase in the sSFR over $z \gtrsim 2$, consistent with more recent derivations (e.g., Bouwens et al. 2012b).

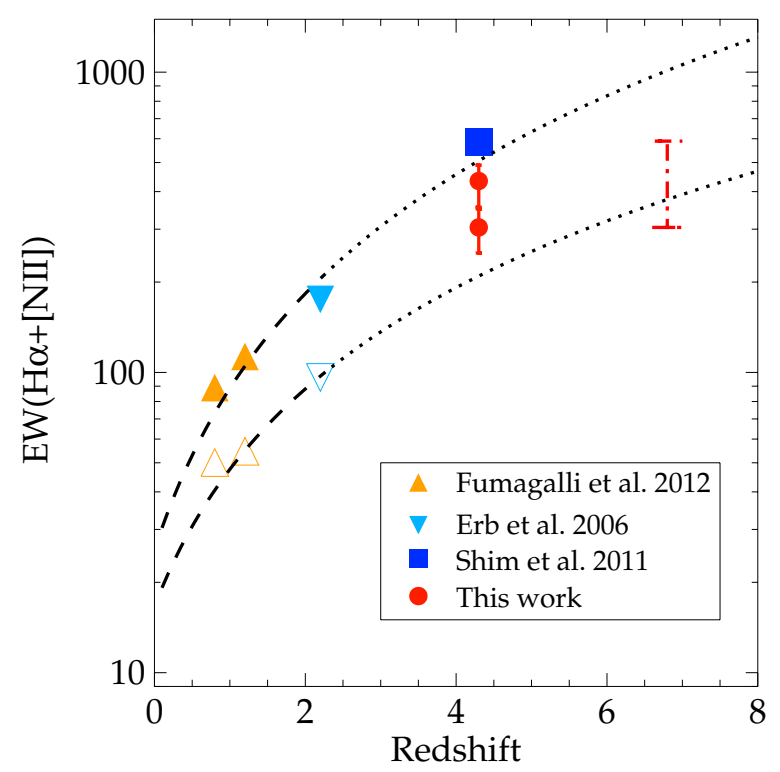

Figure 6. Evolution of the mean $\mathrm{H} \alpha+\left[\mathrm{N}_{\text {II }}\right] \mathrm{EW}$ with redshift. The values at $z<4$ are as compiled in Fumagalli et al. (2012) for star-forming galaxies with stellar mass in the range $\log _{10} M_{\star}=10.0-10.5$. The dashed lines show the power law that Fumagalli et al. (2012) fit to the EW evolution at $z \lesssim 2$, while the dotted lines show an extrapolation of this power law to $z \gtrsim 2$. The open symbols denote the EWs appropriate for the entire galaxy population in Fumagalli et al. (2012), while the solid symbols denote estimates for the starforming subset. The red circles show EWs inferred from the photometric excess method in this paper, with the lower value arising from SED fits including all filters, and the upper value derived from fits excluding the contaminated [3.6] filter (see Section 3.2 for details). The range of EWs illustrated at $z \simeq 6-7$ represents nebular line strengths that we apply to SEDs in Section 4.3, with the lower limit assuming that nebular line strengths remain fixed with increasing redshift and the upper limit assuming they follow a $(1+z)^{1.8}$ power law.

(A color version of this figure is available in the online journal.)

Given these results, it is reasonable to expect nebular lines to be even stronger at $z \simeq 6-7$. Unfortunately, with both IRAC filters contaminated by strong emission lines at these redshifts (Figure 1), we do not have a direct method of estimating the nebular line contamination in this regime. We thus will consider two cases in the following sections. First, we assume conservatively that the nebular line strengths remain fixed at the values derived at $3.8<z<5.0$. While a fixed EW might seem unlikely in light of the power-law evolution at lower redshifts, we note that the rate of increase in the EW might slow if SFHs transition into a phase of rapidly rising SFRs (e.g., Finlator et al. 2011) at $5<z<7$. Second, as a modest upper limit, we consider the case whereby the nebular EWs continue to increase following a $(1+z)^{1.8}$ power law. We conservatively adopt the mean EW of the fitting method including the contaminated [3.6] filter as the $3.8<z<5.0$ reference value for this upper bound.

\subsection{Effect on $z \gtrsim 4$ Stellar Masses}

In Section 4.1, we demonstrated that strong nebular line emission lines make a significant contribution to the broadband flux measurements at $z>3$. If these lines are not accounted for in population synthesis modeling, the rest-optical stellar continuum (and thus the inferred stellar mass and age) will clearly be overestimated (e.g., Schaerer \& de Barros 2010). In principle, these issues can be addressed through nebular+stellar population synthesis models described in Section 3. The drawback of this approach is that the "appropriate" flux from nebular emission for any given model is very uncertain, depending not only on the escape fraction of ionizing radiation but also on the 
reddening law for the nebular gas, both of which are not known at $z>3$.

Here we have attempted to account for these shortcomings using a method that relies on our empirically derived nebular line EW distribution. For each SED we wish to fit, we draw a large number $\left(N \simeq 10^{4}\right)$ of $\mathrm{H} \alpha$ emission line EWs from the distribution we derived in Section 4.1 (e.g., Figure 5, bottom panel). The contribution from [O III] and $\mathrm{H} \beta$ (which contaminate the Spitzer/IRAC filters at $z \gtrsim 5$ ) is obtained by scaling the $\mathrm{H} \alpha$ EW by $1.7-2.0 \times$ (the exact value chosen at random from a uniform distribution), consistent with the flux ratios observed in sub-solar galaxies at $z \simeq 2-3$ (e.g., Hainline et al. 2009; Bian et al. 2010; Erb et al. 2010; Richard et al. 2011a) and those predicted by our nebular+stellar population synthesis models. If the galaxy's redshift places any of these strong lines in the broadband filters we are fitting, we subtract the predicted nebular flux from the photometry.

In order to evaluate how the average properties of the various $z \simeq 4-7$ dropout populations are affected by nebular emission, we consider composite SEDs for the $B-, V-, i^{\prime}$-, and $z$-band dropout populations (e.g., Stark et al. 2009; Labbé et al. 2010b; González et al. 2012) binned by rest-UV magnitude. For the purposes of this section, we limit our analysis to the most recent determinations of the composite SEDs (Labbé et al. 2010b; González et al. 2012), both of which take advantage of WFC3 photometry in the UDF and GOODS fields. The composite rest-optical measurements are constructed by stacking deconfused Spitzer imaging, while the rest-UV averages are obtained through calculation of the median of the individual flux measurements from $H S T$. The number of objects included in the stacks ranges from $>100$ for the $B$-band dropouts to $\simeq 10$ for individual magnitude bins for the $i^{\prime}$-drops. For more details, see González et al. (2012). Since the effect of nebular emission on derived physical properties is clearly redshift dependent (see Figure 1), we must account for the distribution of redshifts within a particular dropout sample. Thus, for each realization of the nebular line EW distribution, we also select a redshift from the expected photometric redshift distribution of the dropout population under consideration (see, e.g., Bouwens et al. 2012b). We fit these realizations of the composite SEDs using the stellar continuum models described in Section 3. Physical properties are determined in a similar manner to that described in Section 3, with uncertainties derived from the $1 \sigma$ intervals of the large number of realizations of the EW distribution.

The impact of nebular emission is clearly seen in the $\log M_{\star}-M_{\mathrm{UV}}$ scaling relations shown in Figure 7 . These are determined for each dropout population with and without the nebular correction from the composite SEDs discussed above. The absolute magnitudes are unchanged in this analysis, so the changes shown are due only to the contamination of broadband light by nebular emission lines. As expected, the impact of nebular emission is strongest in the range $5<z<7$ where nebular lines contaminate both IRAC filters. In contrast, the nebular correction is less severe for the $B$-drop $(z \simeq 4)$ population, since the Spitzer/IRAC $4.5 \mu \mathrm{m}$ filter is devoid of strong emission lines throughout the redshift range covered by $B$-drops (Figure 1).

Assuming that the nebular line EW distribution at $z>5$ remains identical to that determined at $3.8<z<5.0$, we find that the average stellar masses are reduced by factors of $\times 1.1,1.3,1.6$, and 2.4 for the dropout populations centered at $z \simeq 4,5,6$, and 7 , respectively. If the nebular line strengths increase with redshift at $z \gtrsim 5$ following a $(1+z)^{1.8}$ power law (Figure 6; Fumagalli et al. 2012), the typical stellar masses are reduced by $\times 1.9$ and 4.4 at $z \simeq 6$ and 7 , respectively. We emphasize that these represent average corrections applicable to the $B-, V-, i^{\prime}$-, and $z$-band dropout populations. Individual galaxies throughout this redshift range will of course be affected differently.

To summarize, we have used the EW distributions derived in Section 4.1 to compute the likely contribution of nebular emission to broadband photometry. We find that stellar masses at $z \gtrsim 6$ need to be revised downward by $2-4 \times$, with the precise correction depending on whether the EWs of $\mathrm{H} \alpha$ and [O III] emission continue to increase with redshift beyond $z \simeq 5$. This result has an important effect on the $\log M_{\star}-M_{\mathrm{UV}}$ scaling relation, which previously was thought to be largely constant with redshift at $z>4$ (e.g., Stark et al. 2009; González et al. 2011; McLure et al. 2011). It is actually clearly evident in the uncorrected $\log M_{\star}-M_{\mathrm{UV}}$ relations presented in Figure 7 that without nebular corrections, the $M_{\star} / L_{\mathrm{UV}}$ ratios implied by the composite SEDs increase with redshift. Our analysis indicates that this finding is likely to be an artifact of nebular contamination. After correcting for line emission, we demonstrate that the $M_{\star} / L_{\mathrm{UV}}$ ratios are likely to decrease by $\times 1.4-2.5$ with redshift over $4<z<7$. This result has important implications for derivation of the SMD (Section 5.1) and sSFR evolution (Section 5.2) discussed below.

\subsection{Effect of Nebular Continuum Emission}

Nebular continuum emission can also contribute to the observed broadband flux density. Most importantly, the addition of nebular continuum reddens the intrinsic spectrum at young ages, thereby requiring less dust extinction to reproduce the observed colors. This can lead to a reduction in the derived SFRs, in addition to the reduction in the stellar masses discussed earlier. The effects of nebular continuum can be seen in the nebular+stellar models, which typically display spectral edges in the vicinity of the Balmer $/ 4000 \AA$ break (bottom panel of Figure 4). Whether significant nebular continuum is actually contributing to the spectra of high- $z$ galaxies is unclear. Without better sampled SEDs (from, e.g., medium-band near-IR photometry) that might probe such spectral edges, it is difficult to verify the presence of nebular continuum, as we are able to with nebular emission lines. Nevertheless, if emission lines are very strong, it is likely that there is also a significant contribution from nebular continuum emission.

To quantify the likely impact of nebular continuum on the derived physical properties, we examine how the inferred dust reddening and SFR are affected when the nebular continuum is added. To do so, we compare the dust attenuation necessary to reproduce a fixed UV continuum slope for models with and without nebular continuum emission included. Assuming a Calzetti reddening law, we calculate the dust attenuation that reproduces a UV slope with $\beta=-1.5$ as a function of model age. Owing to the redder intrinsic slopes, the inferred dust attenuation is reduced for the nebular+stellar models. The effect is most pronounced at the youngest ages, with the inferred attenuation up to $20 \%$ lower for nebular+stellar models with ages $<30 \mathrm{Myr}$. As a result of the reduced attenuation, a lower normalization is required to match the observed flux density, bringing down the inferred SFRs by up to the $\simeq 20 \%$ level for the youngest systems. In practice, the impact of nebular continuum is more complicated and non-trivial to predict, depending strongly on the shape (i.e., age) of the observed SED. Given these uncertainties, our analysis in the following sections 

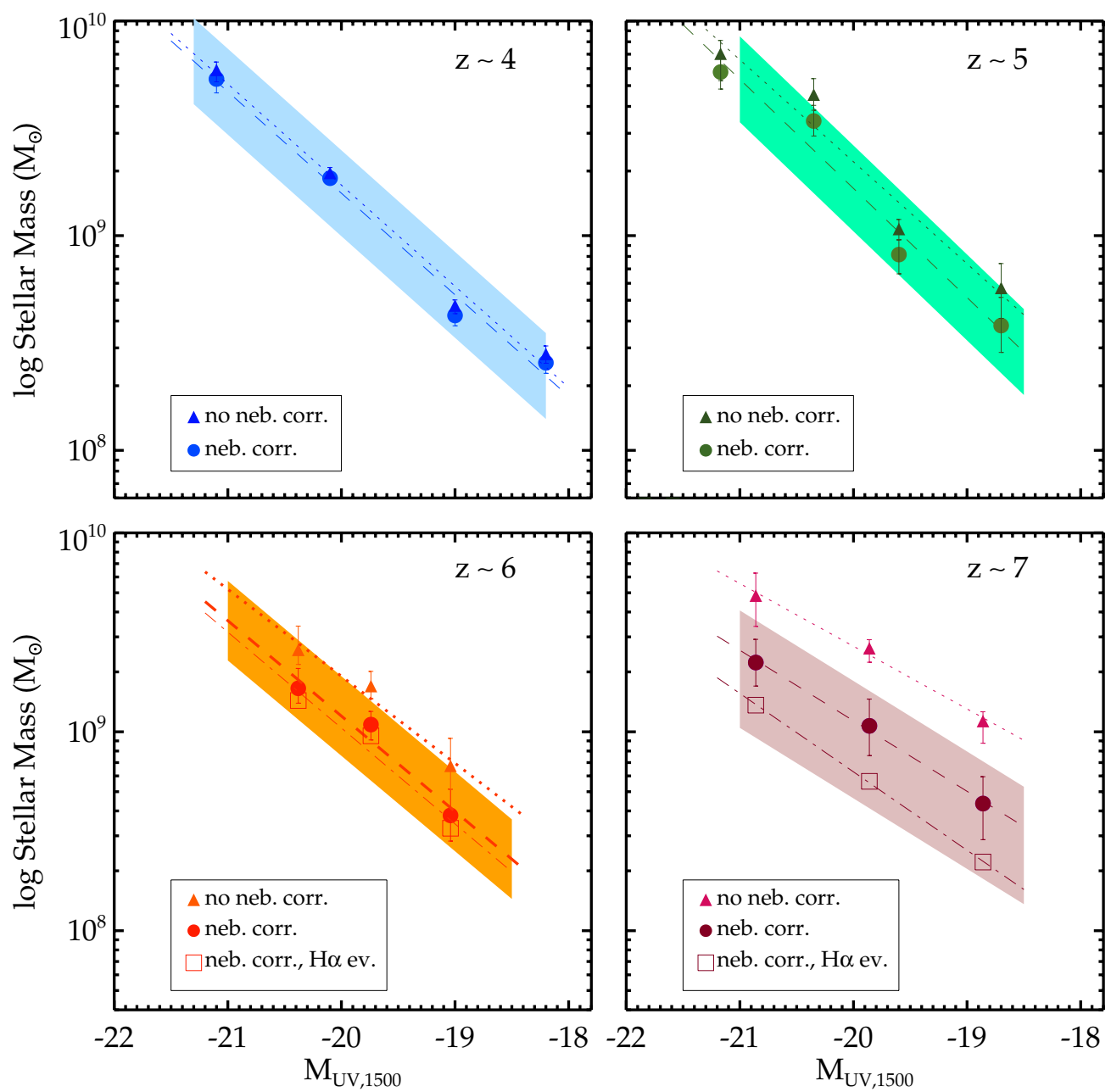

Figure 7. Impact of nebular emission on stellar mass derived using empirical determination of $\mathrm{H} \alpha \mathrm{EW}$ distribution at $3.8<z<5.0$. Solid circles show the $\log M_{\star}-M_{\mathrm{UV}, 1500}$ relationship corrected for nebular contamination of broadband fluxes following the procedure discussed in Section 4.2. The dashed line gives the best linear fit to these data points. Solid triangles show the relationship with no correction for nebular emission assuming that the stellar continuum dominates the broadband flux. Without accounting for nebular emission, the $\log M_{\star}-M_{\mathrm{UV}, 1500}$ relationship does not evolve much with redshift. However, incorporating nebular corrections, the normalization decreases by $1.4-2.5 \times$ over $4<z<7$. The open squares correspond to nebular corrections derived assuming that the EW of nebular emission increases with redshift following the power law shown in Figure 6. The shaded region simply illustrates the range of stellar masses at a given $M_{\mathrm{UV}}$.

(A color version of this figure is available in the online journal.)

will focus on how physical properties are affected by nebular emission lines.

\section{DISCUSSION}

In the previous section, we used the broadband SEDs of a large sample of spectroscopically confirmed galaxies to infer the distribution of nebular line strengths in UV-selected galaxies at $3.8<z<5.0$. We showed that the stellar masses inferred from population synthesis modeling are reduced at $z>5$ when the contribution of these lines to broadband flux densities is removed. In this section, we consider the implications of these results for our current picture of early mass assembly.

\subsection{Stellar Mass Density at $z>3$}

In Section 4.2, we quantified the extent to which the stellar masses of $z>3$ galaxies are affected by nebular emission. Here, we seek to utilize these results to estimate the SMD evolution at $z>3$. To derive the mass densities, we combine the $\log M_{\star}-M_{\mathrm{UV}}$ relationship with UV LFs in a manner mostly similar to that outlined in González et al. (2012). Briefly, we extract a large number $\left(N \simeq 10^{5}\right)$ of luminosities from the measured UV LFs (e.g., Bouwens et al. 2011b). We convert these luminosities to stellar masses using the $\log M_{\star}-M_{\mathrm{UV}}$ relationship and an estimate of the scatter about the median. Whereas earlier studies held the $\log M_{\star}-M_{\mathrm{Uv}}$ relationship fixed with redshift at $z \gtrsim 4$, the strong redshift dependence of nebular contamination (Figure 1) forces us to reconsider the evolution of $M_{\star} / L_{\mathrm{UV}}$ ratios with redshift.

We compute the slope and normalization of the $z \simeq 4$ $\log M_{\star}-M_{\mathrm{UV}}$ relationship using the large sample of LBGs discussed in Stark et al. (2009). For simplicity, we assume that the slope remains constant at $z \gtrsim 4$ and consider only evolution in the normalization of the relationship. To compute the zero points of the $\log M_{\star}-M_{\mathrm{Uv}}$ relation at $z \simeq 5,6$, and 7 , we adjust the measured $z \simeq 4$ relation to account for the relative normalization of the nebular-corrected $\log M_{\star}-M_{\mathrm{UV}}$ relationships shown in Figure 7. To obtain a tentative estimate of the $z \simeq 8 \mathrm{SMD}$, we apply the $z \simeq 7 \log M_{\star}-M_{\mathrm{UV}}$ relationship to the $z \simeq 8 \mathrm{UV}$ LF. In all cases, we use the nebular corrections derived assuming an evolving $\mathrm{H} \alpha$ EW distribution (see Figure 7), but we also discuss how these results would change if the EW distribution remains fixed at $z>5$.

In addition to measurement of the $\log M_{\star}-M_{\mathrm{UV}}$ relationship, accurate determinations of the dispersion are necessary to 

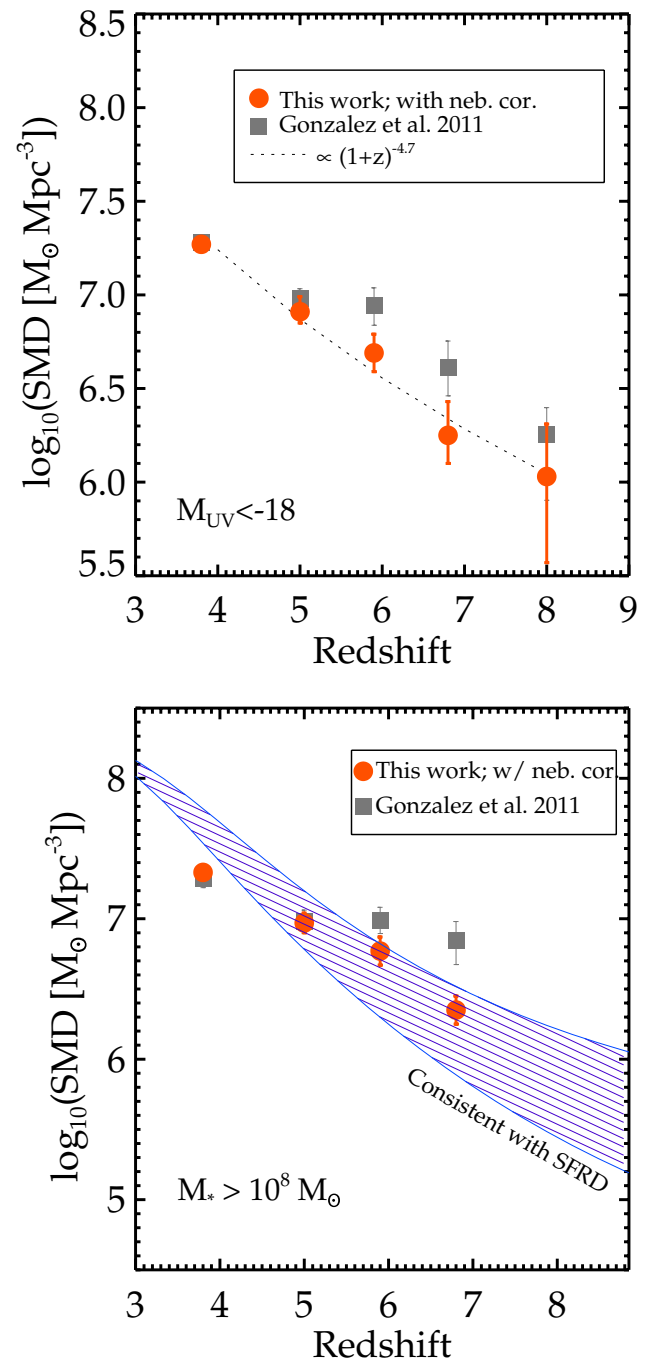

Figure 8. Evolution in the stellar mass density: the mass density is computed by integrating the stellar mass function to a fixed UV luminosity limit (top panel) and fixed stellar mass limit (bottom panel). The main advance with respect to earlier work is the inclusion of corrections for nebular emission contamination of Spitzer/IRAC filters. These are computed using the nebular EW distribution derived from our spectroscopic sample in Section 4.1 and assume that the $\mathrm{H} \alpha$ EW distribution continues to evolve as a power law at $z \gtrsim 5$. The stellar mass functions are derived by combining the nebular-corrected $\log M_{\star}-L_{\mathrm{UV}}$ relation with the UV luminosity functions presented in Bouwens et al. (2012b). We assume that the scatter in the $\log M_{\star}-L_{\mathrm{UV}}$ relation is $\sigma \simeq 0.5$, consistent with previous studies (e.g., González et al. 2011). The blue swath in the bottom panel shows the stellar mass density implied by the evolving star formation rate density, computed by integrating the UV luminosity functions (see Robertson et al. 2010 for details).

(A color version of this figure is available in the online journal.)

account for low-luminosity galaxies with large $M_{\star} / L_{\mathrm{UV}}$ ratios. If scatter is not accounted for, the mass functions will be incomplete and mass densities (above a fixed mass limit) will be underestimated. While a measurement of the observed scatter at $z \simeq 4$ (0.5 dex) was made in González et al. (2011), the intrinsic scatter is likely lower due to systematic uncertainties in the modeling and the effects of nebular contamination. In the following, we assume that the intrinsic scatter is in the range $0.2-0.5$ dex.

We focus first on the UV luminosity limited measure of the SMD, considering only those galaxies with luminosities greater than $M_{\mathrm{UV}}=-18$. The results are presented in Figure 8. As discussed in earlier sections, if nebular emission is not accounted for in the modeling, the inferred $M_{\star} / L_{\mathrm{UV}}$ ratios actually increase moderately with redshift, leading to artificially high SMDs at $z \gtrsim 5$. The inclusion of nebular emission reduces the mass density at $z \simeq 7$ by up to $4 \times$, while having little effect at $z \simeq 4$. As a result of these redshift-dependent corrections, the evolution in the SMD is fit by a steeper power law $\left([1+z]^{-4.7}\right)$ than reported previously.

We also consider the SMD in galaxies with stellar masses in excess of $10^{8} M_{\odot}$. For consistency with earlier measurements of the SMD, the measurements we present in Figure 8 assume that the $\log M_{\star}-M_{\mathrm{UV}}$ relationship has an intrinsic dispersion of $0.5 \mathrm{dex}$. If the scatter is instead only $0.2 \mathrm{dex}$, for example, we would find SMDs that are $\simeq 1.6-2.0 \times$ lower.

While previous estimates of the $6 \lesssim z \lesssim 7$ SMD appeared broadly consistent with the integral of the $z \gtrsim 7$ SFRD (after an appropriate correction for stellar mass loss and recycling), the SMD appeared to be on the high end of the range implied by the SFRD (e.g., Robertson et al. 2010). We compare our revised SMD to those implied by the SFRD in the right panel of Figure 8. The mass density implied by the SFRD is calculated in a similar manner as Robertson et al. (2010), updated to include the latest measurements of the UV LF (Bouwens et al. 2011b). With appropriate corrections for nebular emission, the mass densities now appear in better agreement with the integrated SFRD.

The SMD provides a useful integral constraint on earlier star formation. Spitzer observations of galaxies at $z \simeq 6-7$ therefore offer a valuable measure of the likely ionizing output of galaxies at $7 \lesssim z \lesssim 15$. Therefore, as measurements of the SMD become more reliable, they will offer insight into the contribution of galaxies to reionization, complementing inferences from the UV LF.

We have demonstrated in this paper that corrections for nebular emission are a crucial aspect of obtaining a reliable census of stellar mass in the early universe. In light of the reduced mass density required by nebular contamination, we reconsider the ability of galaxies to achieve reionization by $z \gtrsim 6$, updating the calculation presented in Robertson et al. (2010). Given the consistency with the SFRD (Figure 8), these results are not surprisingly similar to inferences obtained from the UV LF (e.g., Bouwens et al. 2012a; Kuhlen \& FaucherGiguère 2012; Shull et al. 2012). The UV output implied by the mass density is in principle sufficient to achieve reionization by $z \simeq 6-8$ but struggles to account for the optical depth to electron scattering implied by the Wilkinson Microwave Anisotropy Probe (e.g., Larson et al. 2011).

Understanding this photon shortfall will require improved knowledge of how much star formation occurs beyond $z \simeq 10$. While direct detection of $z \gtrsim 10$ galaxies will likely have to wait until JWST, Spitzer offers a unique means of progress in the coming years. By obtaining stellar mass estimates for the emerging samples of $z \simeq 9-10$ galaxies (e.g., Bouwens et al. 2011a; Zheng et al. 2012), it will be possible to obtain some of the first constraints on the contribution of galaxies to the cosmic ionization history beyond $z \simeq 10$.

\section{2. sSFR Evolution}

The reduced stellar masses we infer in Section 4 clearly will affect the evolution of the sSFR at $z>4$. To estimate the impact, we compute the sSFR in fixed stellar mass bins using a similar approach as for the stellar mass function. We draw a large number $\left(N \simeq 10^{5}\right)$ of luminosities from the latest measures of the $z \simeq 4-7$ UV LFs (Bouwens et al. 2012a). For each luminosity and redshift bin, we compute a stellar mass using the $\log M_{\star}-M_{\mathrm{UV}}$ relationship derived in Section 4.1. We consider 
the case in which the strength of nebular emission is constant at $z \gtrsim 4$ and also that in which the emission-line EWs increase with redshift (e.g., Figure 6).

The SFR is computed from $M_{\mathrm{UV}}$ through a series of steps. We account for dust extinction using the UV continuum slopes. For each realization of the UV LF, we draw a UV slope, $\beta$, by adopting the redshift-dependent $\beta-M_{\mathrm{UV}}$ scaling relationships (Bouwens et al. 2012b). The UV slope is then converted to a dust attenuation factor at $1600 \AA$ via the Meurer et al. (1999) IRX- $\beta$ relation $\left(A_{1600}=4.43+1.99 \beta\right)$. The UV luminosity is converted to SFR following the canonical Madau et al. (1998) and Kennicutt (1998) relation $L_{\mathrm{UV}}=\left(\mathrm{SFR} / M_{\odot} \mathrm{yr}^{-1}\right) 8.0 \times$ $10^{27} \mathrm{erg} \mathrm{s}^{-1} \mathrm{~Hz}^{-1}$. This relationship assumes a $0.1-125 M_{\odot}$ Salpeter IMF and constant SFR of $\gtrsim 100$ Myr. Finally, by examining the SFR and $M_{\star}$ of these realizations, we compute the median SSFR of the four dropout samples with stellar mass of $5 \times 10^{9} M_{\odot}$. Before examining the results of this calculation, we discuss two important issues that we have hitherto neglected.

First, we consider how the sSFR is affected by scatter in the $\log M_{\star}-M_{\mathrm{UV}}$ relationship. Note that the sSFR will be overestimated if one merely uses the $\log M_{\star}-M_{\mathrm{UV}}$ relation without taking into account the abundant population of lower SFR objects with large $M_{\star} / L_{\mathrm{UV}}$ ratios. This issue is dealt with in detail in Reddy et al. (2012b) for galaxies at $z \simeq 2-3$. Unfortunately, as we discussed in Section 5.1, the intrinsic scatter is very poorly constrained in UV-selected samples at $z \gtrsim 4$. As a result, previous estimates of the sSFR at $z>4$ have not accounted for $M_{\star} / L_{\mathrm{UV}}$ scatter. To estimate how this shortcoming would affect the sSFR, we add $\log M_{\star}-M_{\mathrm{UV}}$ scatter to the LF realization method described above. This is done by randomly sampling the $M_{\star}$ distribution (defined by the chosen scatter) at a given $M_{\mathrm{UV}}$. If the 0.5 dex observed $z \simeq 4$ scatter reported in González et al. (2011) is entirely intrinsic, then the median sSFR would be reduced by $2.8 \times$ at $z \simeq 4$. Note that one might find slightly different adjustments for the same scatter at other redshifts owing to evolution in the LF. In Section 5.1, we suggested that the intrinsic scatter is likely lower as systematic uncertainties in modeling (including uncertainties in the nebular corrections) surely broaden the dispersion in the stellar masses at fixed UV luminosity. In this case, fewer low-SFR galaxies contribute to the sSFR distribution at fixed mass, resulting in a larger median sSFR. For example, a scatter of 0.2 dex would translate into a reduction of just $1.2 \times$ with respect to the case of no scatter. Physically, one may expect that the scatter at fixed luminosity would increase somewhat between $z \simeq 7$ and $z \simeq 4$, as galaxies have had more time to undergo punctuated episodes of star formation that elevate both their SFRs and mass off of the main sequence. We consider these possibilities in our discussion below.

We now examine a second issue, which pushes the sSFR in the opposite direction. In particular, we examine how scatter (and perhaps systematic offsets) in the conversion between dust-corrected $L_{\mathrm{UV}}$ and SFR affects our sSFR determination. The conversion between UV luminosity and SFR that we use is valid for galaxies with model ages in excess of 100 Myr. Above this age, the conversion factor changes little for an assumed constant SFH. But below $100 \mathrm{Myr}$, a larger SFR is required to produce a fixed $L_{\mathrm{UV}}$ (see Figure 25 of Reddy et al. 2012b). For example, a galaxy with model age of 10 Myr requires a $1.8 \times$ larger SFR to reproduce the same $L_{\mathrm{UV}}$ as a galaxy with 100 Myr. With the reduced ages implied by the nebular corrections, it seems likely that such young systems are present in $z \gtrsim 4$ dropout samples. Inclusion of dispersion in the model
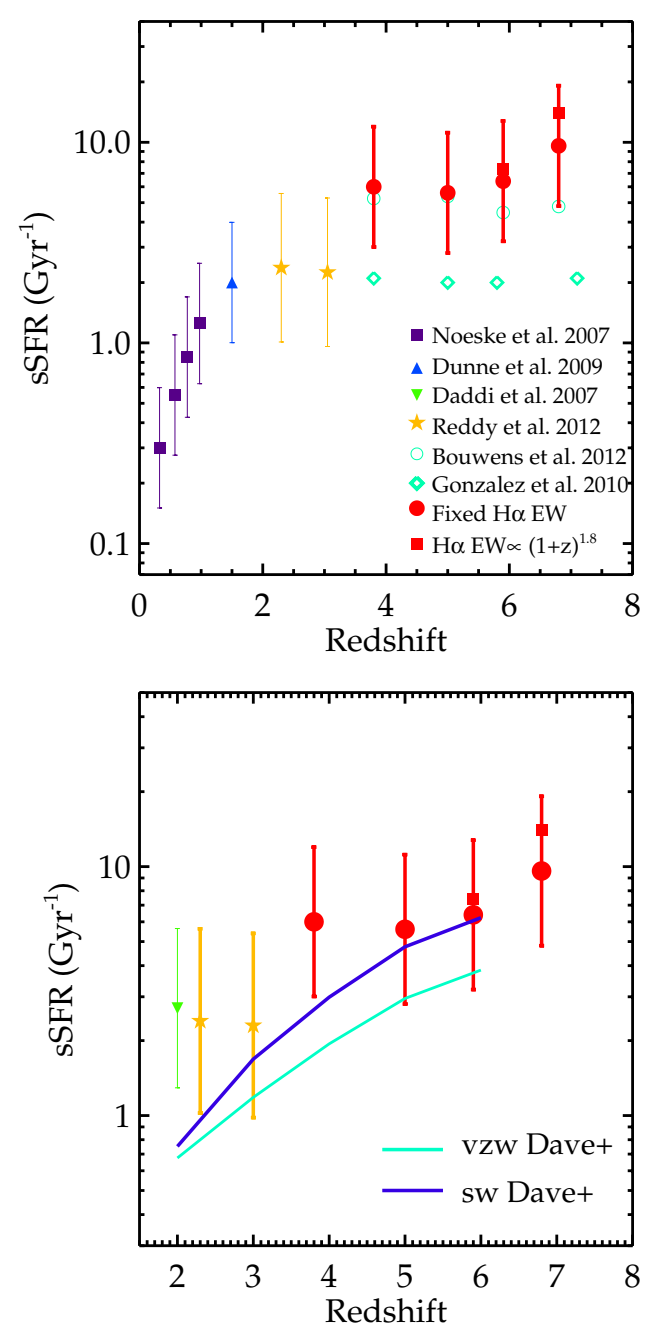

Figure 9. Top: evolution in the sSFR. Our new measurements include stellar masses corrected for nebular emission line contamination. The solid red circles at $z>4$ show values derived assuming that the nebular line EW distribution at $4<z<7$ remains identical to that derived at $3.8<z<5.0$ (Figure 5). The solid squares correspond to values obtained when an evolving nebular line EW distribution is adopted. The error bars show the assumed scatter about the mean sSFR taken from Reddy et al. (2012b). Bottom: comparison of observed sSFR to contemporary theoretical models. The solid lines show the sSFR evolution predicted from cosmological simulations discussed in Davé et al. (2011), with the blue line corresponding to their "slow wind" model and the light green line corresponding to the momentum-driven wind "vzw" model. These models provide adequate fits at the highest redshifts $(z>5)$ but undershoot the observed values at $z \simeq 2-4$.

(A color version of this figure is available in the online journal.)

ages will preferentially shift the median SFR to larger values, resulting in somewhat larger sSFR. Furthermore, it is of course conceivable, if not likely, that such young systems will become more common at higher redshift, requiring a systematic shift toward higher sSFR at earlier times.

We now turn to the derived sSFR evolution, which is shown in the top panel of Figure 9. First, ignoring the effect of nebular emission and the scatter discussed above, we find that the sSFR actually decreases marginally with redshift over $4<z<7$, similar to the findings of Bouwens et al. (2012b). This is driven largely by the redshift dependence of the UV continuum slope $\beta$ versus $M_{\mathrm{UV}}$ relationship. Galaxies at higher redshifts have bluer UV continua (e.g., Bouwens et al. 2012b; Finkelstein et al. 2012), reducing the dust-corrected SFR for a given $M_{\mathrm{UV}}$. Considering the fixed $M_{\star} / L_{\mathrm{UV}}$ ratios assumed in previous 
studies (e.g., Stark et al. 2009; González et al. 2010), it is straightforward to understand this result. As we have discussed above, without nebular corrections, the data actually support a mild increase in the $M_{\star} / L_{\mathrm{UV}}$ ratios with increasing redshift at $z \gtrsim 4$ (Figure 7 ); if the $M_{\star} / L_{\mathrm{UV}}$ ratios were not held fixed (and nebular emission not considered), one would have derived a more rapid decrease in sSFR at $z>4$.

Incorporating our corrections for nebular emission reduces the $M_{\star} / L_{\mathrm{UV}}$ ratios in the $z \simeq 5-7$ LBG samples, increasing the sSFR in this redshift range. If the nebular line EW distribution at $z \gtrsim 5$ is similar to that seen in the bottom panel of Figure 5, we find that the sSFR of galaxies with fixed stellar mass begins to show evidence for positive evolution with redshift, with the $z \simeq 7$ value $\left(9.6 \mathrm{Gyr}^{-1}\right) 4 \times$ larger than that at $z \simeq 2$. This can be viewed as a conservative estimate of the sSFR evolution. As we have argued, however, it is more likely that the EWs of $\mathrm{H} \alpha$ and [O III] increase in strength with redshift at $z \gtrsim 4$, consistent with the evolution seen at intermediate redshift (Fumagalli et al. 2012). Under these assumptions, the derived sSFR shows greater redshift evolution, with the $z \simeq 7$ sSFR $\left(14 \mathrm{Gyr}^{-1}\right)$ roughly $6 \times$ greater than that at $z \simeq 2$ (Reddy et al. 2012b). While intrinsic scatter in the $M_{\star} / L_{\mathrm{UV}}$ ratios might bring these numbers down somewhat (perhaps explaining the excess seen at $z \simeq 4$ ), this is likely counteracted somewhat by scatter and/or systematic evolution in the SFR/ $L_{\mathrm{UV}}$ ratios and possibly a shift toward reduced scatter in the $M_{\star} / L_{\mathrm{UV}}$ ratios at higher redshifts.

To summarize, with the new dust corrections (Bouwens et al. 2012 b) and adjustments for nebular emission contamination, we now find evidence for a power-law increase in the SSFR at $z \gtrsim 2$ that is much more consistent with theoretical expectations than previous observations indicated. Both the absolute values and rate of increase of the sSFR we derive at $z \gtrsim 5$ are very similar to those predicted in the simulations of Davé et al. (2011). de Barros et al. (2012) have also claimed that the sSFR at $z>5$ is greater than previously expected using nebular+stellar modeling of large samples of continuum dropouts. Intriguingly, the sSFR at $2<z<4$ still remains moderately in excess of theoretical expectations. As we have discussed above, the $z \simeq 4$ estimate of the sSFR might come down somewhat owing to scatter in the $M_{\star} / L_{\mathrm{UV}}$. But the $z \simeq 2-3 \mathrm{sSFR}$ measurements include the effects of scatter, so the discrepancy remains puzzling, especially in light of the emerging agreement at $z \gtrsim 5$. Previous theoretical studies have focused on a variety of explanations for the tension at $z \simeq 2$, including a time-varying initial mass function (e.g., Davé 2008; Narayanan \& Davé 2012). Continued efforts along these lines are required to simultaneously explain the high $\mathrm{sSFR}$ at $z \simeq 2$ along with the revised $z \gtrsim 4 \mathrm{sSFR}$ estimates (Figure 9).

Recall that previous indications of a nearly flat sSFR in fixed stellar mass bins at $z>2$ required a mechanism by which star formation is made increasingly inefficient at earlier times. Possibilities included the inefficient formation of molecular hydrogen in metal-poor galaxies (e.g., Robertson \& Kravtsov 2008; Gnedin et al. 2009; Krumholz \& Dekel 2012), or an increase in the mass outflow rate per unit star formation with redshift. The updated estimates of the $z>3$ sSFR no longer obviously require a significant suppression of star formation in galaxies with stellar mass in excess of $10^{9} M_{\odot}$. The current measurements seem consistent with a picture whereby the rapidly increasing baryon accretion rates translate into higher sSFR at earlier times.

As with the SMD, there is room for considerable improvements to these estimates in the coming years. By providing more individual detections of UV-faint galaxies, deeper Spitzer data will enable improved measurements of the slope and scatter of the $\log M_{\star}-M_{\mathrm{UV}}$ relationship in the redshift range considered in Figure 9. It is also of interest to extend these measurements to $z \simeq 8$. In this redshift regime, [O III] lies in the $4.5 \mu \mathrm{m}$ filter, while the $3.6 \mu \mathrm{m}$ filter is devoid of strong lines. Thus, with deep Spitzer data, the SEDs of $z \simeq 8$ systems enable a unique method of deciphering how the strength of nebular emission evolves over $5 \lesssim z \lesssim 8$, one of the key uncertainties in the current analysis.

\section{SUMMARY AND CONCLUSIONS}

Measurements of the evolving stellar mass and sSFR distributions have proven critical to our understanding of early galaxy assembly and the UV photon budget of reionization-era galaxies. Recently, it has become clear that many of these early estimates might be significantly in error due to the contamination of the Spitzer/IRAC bandpasses by nebular emission lines. Knowledge of the strength of these emission lines is necessary for robust determinations of the SMD and sSFR evolution. As these emission lines are shifted out of the observed atmospheric window, direct spectroscopic measurements will not be feasible until JWST.

In this paper, we present a method that enables constraints on nebular emission at $z>4$ by combining large spectroscopic samples and deep Spitzer photometry. Like Shim et al. (2011), we focus on the redshift range $3.8<z<5$.0, over which the IRAC [3.6] filter is contaminated by strong emission lines $(\mathrm{H} \alpha,[\mathrm{NII}],[\mathrm{S} \mathrm{II}])$ while the [4.5] filter is free of nebular contamination. Examining a carefully selected subset of 45 galaxies, we find that the $3.6 \mu \mathrm{m}$ flux is systematically in excess of the expected stellar continuum flux, revealing the presence of strong nebular emission. No excess is seen in a spectroscopic sample at $3.1<z<3.6$, a redshift range over which no strong emission lines contaminate the IRAC filters. We use the photometric excesses in the contaminated [3.6] filter to estimate the $\mathrm{EW}$ distribution of $\mathrm{H} \alpha$ emission at $3.8<z<5.0$. Equipped with this measure of nebular emission at high redshift, we re-evaluate the evolution in the sSFR and SMD at $z \gtrsim 4$. Our primary conclusions from this analysis are summarized below.

1. We find that the mean rest-frame EW of emission lines contaminating the [3.6] filter is $\left\langle\log _{10}\left(W_{3.6} / \AA\right)\right\rangle \simeq 2.57-2.73$. We estimate that $\simeq 76 \%$ of this signal arises from $\mathrm{H} \alpha$, implying an average $\mathrm{H} \alpha \mathrm{EW}$ of $\left\langle\log _{10}\left(W_{\mathrm{H} \alpha} / \AA\right)\right\rangle \simeq 2.45-2.61$ $(280-410 \AA)$ at $3.8<z<5.0$.

2. The mean $\mathrm{H} \alpha \mathrm{EW}$ inferred at $3.8<z<5.0$ appears greater than that for similar star-forming samples at lower redshifts. While definitive knowledge of the evolution in the $\mathrm{H} \alpha$ EW surely awaits direct spectroscopic measurement, the evolution we infer over $2 \lesssim z \lesssim 5$ is certainly consistent with the $(1+z)^{1.8}$ power law derived in Fumagalli et al. (2012). This likely reflects an increase in the sSFR at $z \gtrsim 2$ and importantly suggests that the EW of nebular emission continues to increase at $z \gtrsim 5$.

3. Using the $\mathrm{H} \alpha \mathrm{EW}$ distribution we derive at $3.8<z<5.0$, we explore how nebular contamination is likely to affect the physical properties of galaxies at $z>3$. We find that the stellar masses are reduced, on average, by 1.1, 1.3, 1.6 , and $2.4 \times$ for dropout samples with mean redshifts of $z \simeq 4,5,6$, and 7 , respectively. If the EWs of nebular lines continue to increase in amplitude at $z \gtrsim 5$, we estimate that the reduction in the stellar masses is likely 
to increase to 1.9 and $4.4 \times$ at $z \simeq 6$ and 7 . We note that these corrections are representative for average measures of the dropout populations and not individual galaxies.

4. As the SMD provides a valuable integrated measure of early star formation, constraints on the level of nebular contamination are critical to our knowledge of the ionizing photon budget of galaxies throughout the reionization era. After correcting for nebular emission contamination, we find a factor of $\simeq 2 \times$ reduction from previous estimates. The downward revisions to the SMD improve consistency with expectations from the integrated star formation rate density. Extending such nebular-corrected measurements to emerging galaxy samples at $z \simeq 8-9$ will yield an integral constraint on the UV photon budget during $z \simeq 10-15$.

5. Whereas previous derivations showed little evolution in the sSFR of fixed mass galaxies over $2<z<7$, we demonstrate that after accounting for nebular emission and correcting for dust, the sSFR increases by 4-6x over $2<z<7$. The absolute sSFR values inferred at $z \gtrsim 5$ appear largely similar with predictions from simulations. de Barros et al. (2012) have also recently argued in favor of rapidly rising sSFR at $z>4$ through consideration of models with nebular emission included (see Section 3 for discussion of such models). While there certainly remains room for improvement (in both the data and the modeling), the increase in the SSFR at $z \gtrsim 4$ seems consistent with a picture whereby increasing baryon accretion rates at larger redshift translate into larger sSFR in galaxies of a fixed stellar mass.

We are grateful to Rychard Bouwens, Romeel Davé, Desika Narayanan, Ivo Labbé, Masami Ouchi, Naveen Reddy, Daniel Schaerer, and Valentino González for useful conversations. We thank Romeel Davé for making the results of his simulations available to us. D.P.S. acknowledges support from NASA through Hubble Fellowship grant HST-HF-51299.01 awarded by the Space Telescope Science Institute, which is operated by the Association of Universities for Research in Astronomy, Inc., for NASA under contract NAS5-265555. B.E.R. is partially supported through STScI grant HST-GO-12498.12-A. Support for Program number HST-GO-12498.12-A was provided by NASA through a grant from the Space Telescope Science Institute, which is operated by the Association of Universities for Research in Astronomy, Incorporated, under NASA contract NAS5-26555. J.S.D. acknowledges the support of the European Research Council via the award of an Advanced Grant. J.S.D. and R.J.M. acknowledge the support of the Royal Society via a Wolfson Research Merit Award and a University Research Fellowship, respectively. Some of the data presented herein were obtained at the W. M. Keck Observatory, which is operated as a scientific partnership among the California Institute of Technology, the University of California, and the National Aeronautics and Space Administration. The Observatory was made possible by the generous financial support of the W. M. Keck Foundation.

\section{REFERENCES}

Anders, P., \& Fritze-v. Alvensleben, U. 2003, A\&A, 401, 1063

Bian, F., Fan, X., Bechtold, J., et al. 2010, ApJ, 725, 1877

Bouché, N., Dekel, A., Genzel, R., et al. 2010, ApJ, 718, 1001

Bouwens, R. J., Illingworth, G. D., Franx, M., \& Ford, H. 2007, ApJ, 670, 928

Bouwens, R. J., Illingworth, G. D., Labbe, I., et al. 2011a, Natur, 469, 504

Bouwens, R. J., Illingworth, G. D., Oesch, P. A., et al. 2011b, ApJ, 737, 90
Bouwens, R. J., Illingworth, G. D., Oesch, P. A., et al. 2012a, ApJL, 752, 5 Bouwens, R. J., Illingworth, G. D., Oesch, P. A., et al. 2012b, ApJ, 754, 83 Bruzual, G., \& Charlot, S. 2003, MNRAS, 344, 1000

Calzetti, D., Armus, L., Bohlin, R. C., et al. 2000, ApJ, 533, 682 Curtis-Lake, E., McLure, R. J., Dunlop, J. S., et al. 2012, arXiv:1207.2727 Davé, R. 2008, MNRAS, 385, 147

Davé, R., Finlator, K., \& Oppenheimer, B. D. 2012, MNRAS, 421, 98 Davé, R., Oppenheimer, B. D., \& Finlator, K. 2011, MNRAS, 415, 11 Dayal, P., \& Ferrara, A. 2012, MNRAS, 421, 2568 de Barros, S., Schaerer, D., \& Stark, D. P. 2012, arXiv:1207.3663 Dekel, A., Birnboim, Y., Engel, G., et al. 2009, Natur, 457, 451 Dutton, A. A., van den Bosch, F. C., \& Dekel, A. 2010, MNRAS, 405, 1690 Egami, E., Kneib, J.-P., Rieke, G. H., et al. 2005, ApJL, 618, 5 Erb, D. K., Pettini, M., Shapley, A. E., et al. 2010, ApJ, 719, 1168 Erb, D. K., Steidel, C. C., Shapley, A. E., et al. 2006, ApJ, 647, 128 Eyles, L. P., Bunker, A. J., Ellis, R. S., et al. 2007, MNRAS, 374, 910 Eyles, L. P., Bunker, A. J., Stanway, E. R., et al. 2005, MNRAS, 364, 443 Faber, S. M., Phillips, A. C., Kibrick, R. I., et al. 2003, Proc. SPIE, 4841, 1657 Fazio, G. G., Ashby, M. L. N., Barmby, P., et al. 2004, ApJS, 154, 39 Finkelstein, S. L., Papovich, C., Salmon, B., et al. 2012, ApJ, 756, 164 Finlator, K., Oppenheimer, B. D., \& Davé, R. 2011, MNRAS, 410, 1703 Förster Schreiber, N. M., Genzel, R., Bouché, N., et al. 2009, ApJ, 706, 1364 Fumagalli, M., Patel, S., Franx, M., et al. 2012, ApJL, 757, 22

Giavalisco, M., Ferguson, H. C., Koekemoer, A. M., et al. 2004, ApJL, 600, 93 Gnedin, N. Y., Tassis, K., \& Kravtsov, A. V. 2009, ApJ, 697, 55 González, V., Bouwens, R., Labbe, I., et al. 2012, ApJ, 755, 148 González, V., Labbé, I., Bouwens, R. J., et al. 2010, ApJ, 713, 115 González, V., Labbé, I., Bouwens, R. J., et al. 2011, ApJL, 735, 34 Gordon, K. D., \& Clayton, G. C. 1998, ApJ, 500, 816

Grazian, A., Fontana, A., de Santis, C., et al. 2006, A\&A, 449, 951

Grogin, N. A., Kocevski, D. D., Faber, S. M., et al. 2011, ApJS, 197, 35 Hainline, K. N., Shapley, A. E., Kornei, K. A., et al. 2009, ApJ, 701, 52 Jones, T., Stark, D. P., \& Ellis, R. S. 2012, ApJ, 751, 51

Kennicutt, R. C., Jr. 1998, ARA\&A, 36, 189

Koekemoer, A. M., Faber, S. M., Ferguson, H. C., et al. 2011, ApJS, 197, 36 Krumholz, M. R., \& Dekel, A. 2012, ApJ, 753, 16

Kuhlen, M., \& Faucher-Giguère, C.-A. 2012, MNRAS, 423, 862

Labbé, I., Bouwens, R., Illingworth, G. D., \& Franx, M. 2006, ApJL, 649, 67

Labbé, I., González, V., Bouwens, R. J., et al. 2010a, ApJL, 708, 26

Labbé, I., González, V., Bouwens, R. J., et al. 2010b, ApJL, 716, 103

Larson, D., Dunkley, J., Hinshaw, G., et al. 2011, ApJS, 192, 16

Lee, J. C., Kennicutt, R. C., Funes, S. J., et al. 2007, ApJL, 671, 113

Lee, J. C., Ly, C., Spitler, L., et al. 2012, PASP, 124, 782

Lee, K.-S., Ferguson, H. C., Wiklind, T., et al. 2012, ApJ, 752, 66

Ly, C., Lee, J. C., Dale, D. A., et al. 2011, ApJ, 726, 109

Madau, P., Pozzetti, L., \& Dickinson, M. 1998, ApJ, 498, 106

Maiolino, R., Nagao, T., Grazian, A., et al. 2008, A\&A, 488, 463

Mancini, C., Förster Schreiber, N. M., Renzini, A., et al. 2011, ApJ, 743, 86

McLure, R. J., Dunlop, J. S., de Ravel, L., et al. 2011, MNRAS, 418, 2074

Meurer, G. R., Heckman, T. M., \& Calzetti, D. 1999, ApJ, 521, 64

Narayanan, D., \& Davé, R. 2012, MNRAS, 423, 3601

Neistein, E., \& Dekel, A. 2008, MNRAS, 383, 615

Nestor, D. B., Shapley, A. E., Steidel, C. C., \& Siana, B. 2011, ApJ, 736, 18

Oke, J. B., \& Gunn, J. E. 1983, ApJ, 266, 713

Ono, Y., Ouchi, M., Shimasaku, K., et al. 2010, ApJ, 724, 1524

Onodera, M., Arimoto, N., Daddi, E., et al. 2010, ApJ, 715, 385

Osterbrock, D. E., \& Ferland, G. J. (ed.) 2006, Astrophysics of Gaseous Nebulae and Active Galactic Nuclei (2nd ed.; Sausalito, CA: Univ. Science Books)

Papovich, C., Finkelstein, S. L., Ferguson, H. C., Lotz, J. M., \& Giavalisco, M. 2011, MNRAS, 412, 1123

Reddy, N., Dickinson, M., Elbaz, D., et al. 2012a, ApJ, 744, 154

Reddy, N. A., Erb, D. K., Pettini, M., Steidel, C. C., \& Shapley, A. E. 2010, ApJ, 712, 1070

Reddy, N. A., Pettini, M., Steidel, C. C., et al. 2012b, ApJ, 754, 25

Retzlaff, J., Rosati, P., Dickinson, M., et al. 2010, A\&A, 511, A50

Richard, J., Jones, T., Ellis, R., et al. 2011a, MNRAS, 413, 643

Richard, J., Kneib, J.-P., Ebeling, H., et al. 2011b, MNRAS, 414, L31

Robertson, B. E., Ellis, R. S., Dunlop, J. S., McLure, R. J., \& Stark, D. P. 2010, Natur, 468, 49

Robertson, B. E., \& Kravtsov, A. V. 2008, ApJ, 680, 1083

Salpeter, E. E. 1955, ApJ, 121, 161

Santini, P., Fontana, A., Grazian, A., et al. 2009, A\&A, 504, 751

Schaerer, D., \& de Barros, S. 2009, A\&A, 502, 423

Schaerer, D., \& de Barros, S. 2010, A\&A, 515, A73

Schenker, M. A., Stark, D. P., Ellis, R. S., et al. 2012, ApJ, 744, 179

Shapley, A. E. 2011, ARA\&A, 49, 525

Shim, H., Chary, R.-R., Dickinson, M., et al. 2011, ApJ, 738, 69 
Shull, J. M., Harness, A., Trenti, M., \& Smith, B. D. 2012, ApJ, 747, 100

Siana, B., Teplitz, H. I., Chary, R.-R., Colbert, J., \& Frayer, D. T. 2008, ApJ, 689,59

Stark, D. P., Bunker, A. J., Ellis, R. S., Eyles, L. P., \& Lacy, M. 2007, ApJ, 659,84

Stark, D. P., Ellis, R. S., Bunker, A., et al. 2009, ApJ, 697, 1493

Stark, D. P., Ellis, R. S., Chiu, K., Ouchi, M., \& Bunker, A. 2010, MNRAS, 408, 1628

Stark, D. P., Ellis, R. S., \& Ouchi, M. 2011, ApJL, 728, 2

Trenti, M., Stiavelli, M., Bouwens, R. J., et al. 2010, ApJL, 714, 202
Trump, J. R., Weiner, B. J., Scarlata, C., et al. 2011, ApJ, 743, 144

Vanzella, E., Giavalisco, M., Dickinson, M., et al. 2009, ApJ, 695, 1163

Vanzella, E., Giavalisco, M., Inoue, A. K., et al. 2010, ApJ, 725, 1011

Wang, W.-H., Cowie, L. L., Barger, A. J., Keenan, R. C., \& Ting, H.-C. 2010, ApJS, 187, 251

Weinmann, S. M., Neistein, E., \& Dekel, A. 2011, MNRAS, 417, 2737

Windhorst, R. A., Cohen, S. H., Hathi, N. P., et al. 2011, ApJS, 193, 27

Wuyts, S., Förster Schreiber, N. M., Lutz, D., et al. 2011, ApJ, 738, 106

Zheng, W., Postman, M., Zitrin, A., et al. 2012, Natur, 489, 406 